GENERALISATIONS OF QUASI-HYPERBOLIC DISCOUNTING

BY

\title{
SHANELLA RAJANAYAGAM
}

\begin{abstract}
A thesis
submitted to the Victoria University of Wellington

in fulfilment of the requirements for the degree of

Master of Commerce
\end{abstract}

Victoria University of Wellington

2016 


\section{Abstract}

This paper proposes several time preference specifications that generalise quasi-hyperbolic discounting, while retaining its analytical tractability. We define their discount functions and provide a recursive formulation of the implied lifetime payoffs. A calibration exercise demonstrates that these specifications deliver better approximations to true hyperbolic discounting. We characterise the Markov-perfect equilibrium of a general intra-personal game of agents with various time preferences. When applied to specific economic examples, our proposals yield policies that are close to those of true hyperbolic discounters. Furthermore, these approximations can be used in settings where an exact solution for hyperbolic agents is not available. Finally, we suggest further generalisations which would provide an even better fit.

KEYWORDS: hyperbolic discounting, approximations, intra-personal games 


\section{Introduction}

Decision-making often involves trade-offs between costs and benefits at different points in time. To explore these trade-offs and gain insight into intertemporal choices, economic theory introduces the concept of time preferences. The existing literature has considered numerous approaches to evaluating and comparing payoffs in dynamic settings. My paper contributes to this literature by proposing time preference specifications which deliver a better fit to experimental and empirical evidence while retaining analytical tractability.

The most popular framework for analysing intertemporal problems is that of discounted utility theory (Samuelson, 1937). This approach is also known as "exponential discounting". It has been very popular in economic research, as it offers analytical convenience and yields optimal plans that are time consistent. However, there are many situations in which human behaviour cannot be explained with exponential discounting. In fact, multiple studies agree that, in the real world, agents' preferences exhibit "present bias" or "increasing patience". In particular, people are more patient regarding intertemporal trade-offs that are further in the future. Such preferences imply a discrepancy between the objectives of the current decision maker and his future selves. As a result, the plan that is currently deemed optimal would be subject to change if commitment technologies are not available. In such an environment, the ability to commit to future actions is valuable, as it can increase lifetime payoffs.

There is vast evidence in support of increasing patience. In experiments, this phenomenon often manifests as "preference reversal": subjects change their preferences with the advancement of time. Chung and Herrnstein (1961) observed that some aspects of animal behaviour are consistent with present bias. Their results were later extended to human subjects as well (see Ainslie (1992) for a survey). These experiments have been carried out with a wide range of real rewards. For example, Millar and Navarick (1984) studied the effects of videogames as a positive reinforcer. 
They performed two experiments involving a choice of selecting between longer or shorter periods of access to a videogame. The first experiment showed that immediate reinforcement was preferred to delayed reinforcement while, in the second, preference for the shorter delay decreased, possibly due to "preference reversal". Christensen-Szalanski (1984) examined the attitudes of 18 pregnant women toward avoiding pain and avoiding anesthesia. The women preferred to avoid using anesthesia during childbirth when asked one month before labour and during early labour. However, during active labour their preferences shifted toward avoiding pain. Green, Fry and Myerson (1994) studied three age groups: children, young adults and older adults. Subjects in each age group had to choose between immediate and delayed hypothetical monetary rewards. The amount of the delayed reward was held constant while its delay was varied. All three age groups showed delayed discounting, with the rate of discounting being highest for children and lowest for older adults. For further experimental research on time discounting see Frederick, Loewentstein and O'Donoghue (2002).

Over the years, there have been multiple proposals of discounting models that incorporate increasing patience (Ainslie, 1975; Mazur, 1987). Probably the most general specification was suggested by Loewenstein and Prelec (1992). It captures the other proposals as special cases, and is thus referred to as "generalised hyperbolic discounting". Moreover, Loewenstein and Prelec are able to provide axiomatic foundations for their approach. They postulate that the delay necessary to compensate a decision maker for a larger outcome is a linear function of the time to a smaller, earlier outcome. The authors show that this assumption is satisfied only by the generalised hyperbolic discount function.

While true hyperbolic preferences provide a good fit to real-world data and experimental evidence, they do pose substantial analytical challenges. Specifically, they are not amenable to analysis with standard techniques such as dynamic programming. Moreover, lifetime payoffs of agents with 
such preferences are often infinite, implying a lack of interior solutions. To avoid these difficulties, the existing literature often assumes an alternative specification knows as "quasi-hyperbolic discounting". It was first proposed by Phelps and Pollak (1968) in the context of an inter-generational bequest game. Later, Laibson used these preferences to approximate true hyperbolic discounting (Laibson, 1997; Laibson, 1998).

In essence, quasi-hyperbolic discounting specifies a lower discount factor only for the trade-off between today and tomorrow. For all other future adjacent trade-offs, the discount factor is constant from the current point of view. The quasi-hyperbolic formulation is analytically convenient, as it permits recursive formulation of intertemporal problems. Furthermore, it does exhibit a form of increasing patience: agents with these preferences are more impatient about immediate tradeoffs (i.e. today versus tomorrow) as opposed to trade-offs that will happen in the distant future. However, a shortcoming of quasi-hyperbolic discounting is that it gives rise a discontinuous break between the "short run" and the "long run". That is, it does not generate a discount factor that is gradually increasing over time. Instead, discounting effectively becomes exponential from tomorrow onward.

In this paper, we build on the pioneering contribution of Phelps and Pollak (1968) and Laibson (1997) by proposing several generalisations of quasi-hyperbolic discounting. These generalisations still allow us to formulate the decision makers' problems recursively, and thus to characterise intertemporal choices with standard dynamic programming techniques. In addition, our formulations nest quasi-hyperbolic preferences as a special case. This guarantees that they will always provide better approximations to true hyperbolic discounting. Our first proposal yields an effective discount factor that increases over the first two consecutive trade-offs, whilst our second proposal exhibits decreasing impatience over the entire planning horizon. The superior goodness of fit of these approximations is demonstrated with a calibration exercise. 
We then show that our generalisations may have non-trivial repercussions for several economic applications. To do so, we first study the Markov-perfect equilibrium (MPE) of a general intrapersonal game between the current and future selves of a sophisticated decision maker. We apply the analysis of this general setting to two specific economic problems: i) Laibson's consumptionsaving model (Laibson, 1998) and ii) smoking and internality taxes (Gruber and Koszegi, 2001). In these applications, the equilibria with true hyperbolic discounters can be solved for explicitly (Calcott and Petkov, 2015). We compare them to the equilibria that would arise under the various approximations of true hyperbolic discounting, and show that our proposals yields policies that are much closer to the benchmark.

Next we consider two extensions in which the analysis of Calcott and Petkov (2015) is not applicable. In the first, consumer utility incorporates Markovian uncertainty and in the second, payoffs are linear-quadratic. These settings violate the assumptions of Calcott and Petkov (2015). Therefore, it is not possible to obtain exact solutions for agents with true hyperbolic preferences. However, when used in these frameworks, our generalisations would still yield interior solutions. We illustrate these solutions with numerical examples.

Finally, we show that our specifications can be generalised further, thus enabling us to attain an even better approximation to true hyperbolic discounting. However, higher precision comes at the expense of increased analytical and computational complexity. We describe four such generalisations, and derive the corresponding Euler equations of the decision maker's intra-personal game.

The remainder of this thesis is organised as follows. In Section 2 we provide a brief overview of time preferences that are popular in the existing literature, i.e. exponential, true hyperbolic and quasi-hyperbolic. In Section 3 we propose our first generalisation. We define its discount function and provide a recursive formulation of the implied lifetime payoffs. In Section 4 we introduce 
our second generalisation. We first define it recursively, and then obtain a closed-form solution for its discount function. Again, we are able to formulate lifetime payoffs recursively. Section 5 presents a calibration exercise. We assume that actual discounting is true hyperbolic, and choose the parameters of the various quasi-hyperbolic approximations to deliver the best fit. The exercise demonstrates that our proposals yield better approximations. In Section 6 we study a general intra-personal game with linear state dynamics, and derive equilibrium conditions for agents with true and quasi-hyperbolic preferences. This analysis is applied to two economic problems in Section 7. We compute their equilibria and show that, when calibrated, our generalisations deliver policies that are much closer to those implied by true hyperbolic discounting. Section 8 explores a couple of extensions in which an exact solution for true hyperbolic discounters is not available, but we can still use our approximations. Section 9 shows how to generalise our proposals further to attain an even better fit. Finally, Section 10 concludes the thesis.

\section{Time preferences considered in the literature}

Time preferences can be summarised succinctly with a discount function $D(\tau): N \rightarrow[0,1]$. A period- $t$ agent who expects to receive a payoff $u^{\tau+t}$ in period $\tau+t$ would assign this payoff a current value of $D(\tau) u^{\tau+t}$. If faced with a stream of instantaneous payoffs $u^{t}, u^{t+1}, u^{t+2}, \ldots$, the agent's lifetime payoff from the perspective of period $t$ could be expressed as

$$
D(0) u^{t}+\sum_{\tau=1}^{\infty} D(\tau) u^{t+\tau} \text {. }
$$

It is assumed that $D(0)=1$, and that $D(\tau)$ is decreasing in $\tau$ and converging to 0 as $\tau$ approaches infinity.

The economic interpretation of the discount function is that it reflects the impatience of the 
decision maker. Note that his effective discount factor for a trade-off between two successive periods, $\tau+t$ and $\tau+t+1$, would be given by $D(\tau+1) / D(\tau) \in(0,1)$. If this ratio is close to zero, this means that the period- $\tau+t$ payoff is much more important to the decision maker than the period- $\tau+t+1$ payoff. If, on the other hand, $D(\tau+1) / D(\tau)$ is close to one, then he assigns equal priority to the two periods.

\subsection{Exponential discounting}

The standard discounted utility model was proposed by Samuelson (1937). He considered the following discount function:

$$
D_{0}(\tau)=\delta^{\tau}
$$

Hence, the lifetime payoff of a period- $t$ decision maker with these preferences is

$$
W^{t}=u^{t}+\sum_{\tau=1}^{\infty} \delta^{\tau} u^{\tau+t}
$$

This type of discounting is known as exponential discounting. A special feature of this formulation is that, for any $\tau$, we have $D_{0}(\tau+1) / D_{0}(\tau)=\delta$.

Exponential discounting has been very popular in the literature for two reasons.

- First, it offers analytical convenience, as it allows for simple recursive formulation of intertemporal problems. In particular, the lifetime payoff of a period-t agent can be expressed as

$$
W^{t}=u^{t}+\delta W^{t+1} \text {. }
$$

On the basis of this representation, Bellman (1952) developed the theory of dynamic programming. Using a contraction mapping argument, they were able to prove existence and 
uniqueness of solutions to problems involving intertemporal trade-offs.

- Second, Strotz (1955-1956) showed that only exponential discounting produces optimal plans that are dynamically consistent. In particular, suppose that an agent is able to recalculate his optimal plan in a future period subsequent to $t$. If he does not have an incentive to deviate from the plan originally devised in period $t$, then this plan is said to be dynamically consistent. To satisfy this property, the trade-off between two adjacent periods should be independent of the agent's viewpoint. That is, $D(\tau+1) / D(\tau)=D(t+\tau+1) / D(t+\tau)$ for any $\tau, t$. The only discount function which meets this requirement is $D_{0}(\tau)$.

\subsection{True hyperbolic discounting}

The experimental and empirical literature has failed to find extensive support for exponential preferences. In fact, numerous studies show that there are many types of behaviour which cannot be explained by exponential discounting. For example, one commonly observed phenomenon is known as "preference reversal". That is, people often prefer $\$ 100$ today to $\$ 110$ tomorrow, but $\$ 110$ in day 31 is preferred to $\$ 100$ in day 30 . Such choices cannot be reconciled with exponential discounting. Another example is provided by Thaler (1981). Participants in an experiment were asked how much money is required in 1 month, 1 year and 10 years to make them indifferent to receiving $\$ 15$ today. The median answers of the subjects were $\$ 20, \$ 50$ and $\$ 100$. Again, these answers would imply discount factors that change dramatically with the agent's perspective.

Most of the papers on time preferences argue that the observed data can be explained better if agents are assumed to have true hyperbolic discounting instead of exponential discounting. The true hyperbolic discount function was originally defined by Ainslie and Herrnstein (1981) and Mazur (1987) as follows:

$$
D_{h}(\tau)=\frac{1}{1+d \tau}
$$


The parameter $d$ reflects the agent's impatience: the higher $d$, the lower the weight on future payoffs. Later this function was given a more general form, $D_{h}^{\prime}(\tau)=(1+d \tau)^{-a / d}$, and was justified axiomatically by Loewenstein and Prelec (1992). A key feature of these formulations is that the discount factor $D(\tau+1) / D(\tau)$ between two adjacent periods is gradually increasing in $\tau$ :

$$
D\left(\tau^{\prime}+1\right) / D\left(\tau^{\prime}\right)>D\left(\tau^{\prime \prime}+1\right) / D\left(\tau^{\prime \prime}\right) \text { for all } \tau^{\prime}>\tau^{\prime \prime}
$$

This feature is known as present bias. ${ }^{1}$

As pointed out earlier, non-stationarity of the effective discount factors may imply that the optimal precommitment plan of a hyperbolic decision maker is time inconsistent. In the absence of commitment technologies, future agents will be tempted to deviate from the plan that was previously considered optimal. In other words, this decision maker will have a self-control problem. The literature has considered several approaches to modelling the behaviour of such decision makers. Our analysis will focus exclusively on sophisticated agents. That is, we assume that agents are aware of future temptations to deviate and take actions to mitigate them. This view is consistent with several economic phenomena that are observed in the real world. We will discuss these in detail in Section 7. Technically, the behaviour of a sophisticated decision maker is modelled as an intrapersonal game between his current and future selves. The subgame-perfect equilibrium of this game yields a dynamically consistent decision stream.

While true hyperbolic discounting can explain empirical observations, it poses significant analytical challenges. Specifically, it does not allow for a recursive representation of dynamic problems. That is, formulating an equation that defines an agent's lifetime payoff through itself is infeasible. Moreover, representing these payoffs with value functions in infinite-horizon settings is problematic.

\footnotetext{
${ }^{1}$ Note that exponential discounters are not present-biased, as their effective discount factor is always constant.
} 
The effective discount factor between adjacent periods implied by (2) approaches 1 as the length of the planning horizon increases: $\lim _{\tau \rightarrow \infty} D_{h}(\tau+1) / D_{h}(\tau)=1$. This suggests that lifetime payoffs are typically infinite. ${ }^{2}$

\subsection{Order-one quasi-hyperbolic discounting}

The existing literature often circumvents the aforementioned technical difficulties by adopting an alternative specification known as $(\beta, \delta)$ discounting (or quasi-hyperbolic discounting). To distinguish it from our generalisations, we will refer to it as "order-one quasi-hyperbolic discounting". This specification is a simple way of capturing the idea that the discount factor for (at least some) adjacent periods may be increasing as trade-offs become more distant in time. Last but not least, these preferences are analytically convenient: they allow us to formulate intertemporal problems recursively.

Quasi-hyperbolic discounting was first introduced by Phelps and Pollak (1968) in the context of inter-generational preferences. Later it was used by Laibson (1997) to study the savings behaviour of a consumer with self-control problems who has access to imperfect commitment devices (e.g. illiquid assets). Laibson's key finding was that quasi-hyperbolic discounting will cause consumers to undersave: each subsequent self will consume too much from the current self's point of view. All intertemporal selves can be made better off if each of them agreed to save more. Quasi-hyperbolic preferences have also been applied to the problems of procrastination (O'Donoghue and Rabin, 1999), retirement decisions (Diamond and Koszegi, 2003), asset pricing (Kocherlakota, 2001), job search (Paserman, 2008), growth (Barro, 1999) and addiction (Gruber and Koszegi, 2001).

\footnotetext{
${ }^{2}$ Recently Calcott and Petkov (2015) have managed to obtain an explicit solution for true hyperbolic agents with a specific utility function. However, their analysis can be used in a narrow class of models. In contrast, our approach can be applied outside the range of these settings. For more detail see Section 8 .
} 


\subsubsection{Definition}

In essence $(\beta, \delta)$ preferences specify a lower discount factor only for the trade-off between the current period and the one that follows immediately. For any other two consecutive future periods, the discount factor is higher and invariant to the agent's perspective. Formally, the discount function is defined as

$$
D_{1}(0)=1, \quad D_{1}(\tau)=\beta \delta^{\tau} \text { for } \tau \geqslant 1
$$

The parameter $\beta$ is usually assumed to be in the interval $(0,1] .{ }^{3}$ From the viewpoint of period $t$, the decision maker's lifetime utility is thus given by:

$$
V^{t}=u^{t}+\beta \sum_{\tau=1}^{\infty} \delta^{\tau} u^{\tau+t}
$$

The preferences are time invariant. When period $t+1$ arrives, lifetime utility from the perspective of that period will be:

$$
V^{t+1}=u^{t+1}+\beta \sum_{\tau=1}^{\infty} \delta^{\tau} u^{\tau+t+1}
$$

The above definition specifies a discount factor of $\beta \delta$ for the trade-off between today and tomorrow. However, for any other two adjacent time periods, $\tau+1$ and $\tau, \tau \geqslant 1$, we have $D_{1}(\tau+1) / D_{1}(\tau)=\delta$. The implication is that order-one quasi-hyperbolic discounting partially captures an important qualitative feature of true hyperbolic discounting: agents with such preferences are more impatient about trade-offs that are closer to their viewpoint. However, unlike true hyperbolic discounting, (3) does not give rise to a discount factor that is gradually increasing over the entire planning horizon. From next period on, order-one quasi-hyperbolic discounting effectively becomes exponential. This feature substantially simplifies the analysis of economic problems, but

\footnotetext{
${ }^{3}$ When $\beta=1, D_{1}(\tau)$ reduces to the standard exponential discount function.
} 
also detracts from their realism. Indeed, few studies have found that $(\beta, \delta)$ preferences provides a good fit to observed data.

\subsubsection{Recursive representation of payoffs}

Quasi-hyperbolic discounting is assumed primarily for analytical convenience, as it allows us to formulate dynamic problems recursively. That is, we are able to represent an agent's lifetime payoff as an expression of itself. The recursive formulation enables us to use dynamic programming in order to characterise the time-consistent equilibrium in dynamic problems of agents with non-exponential time preferences.

The recursive formulation for $(\beta, \delta)$ discounting was analysed in detail by Laibson (1997). We now present a brief overview of his approach. The lifetime payoff $U^{t}$ of the decision maker's period- $t$ self is as defined by (4). Let $W^{t+1}$ be the continuation payoff, i.e. the present value of all payoffs he will receive after today. As noted earlier, the period- $t$ agent's preferences effectively become exponential from period $t+1$ onward, implying that

$$
W^{t+1}=u^{t+1}+\sum_{\tau=1}^{\infty} \delta^{\tau} u^{\tau+t+1}
$$

Consequently, we can express $V^{t}$ as

$$
V^{t}=u^{t}+\beta \delta W^{t+1}
$$

Since $W^{t+1}$ is just the lifetime payoff of an exponential discounter, it can be defined recursively as in Subsection 2.1:

$$
W^{t+1}=u^{t+1}+\delta W^{t+2} .
$$


Moreover, it is possible to derive an equation that links $V^{t}$ and $W^{t}$. By definition,

$$
W^{t}=u^{t}+\sum_{\tau=1}^{\infty} \delta^{\tau} u^{\tau+t}
$$

Multiplying (7) by $\beta$ and subtracting it from (4) yields

$$
V^{t}=(1-\beta) u^{t}+\beta W^{t}
$$

\section{Order-two quasi-hyperbolic discounting}

This section describes a proposal for an alternative specification of time preferences which is also amenable to recursive representation. We will refer to it as "order-two quasi-hyperbolic". As before, we will provide a recursive formulation of the lifetime payoff on an agent with such preferences. The complexity of the analysis increases only slightly relative to that in the previous section.

\subsection{Definition of the discount function}

Our first proposal generalises order-one quasi-hyperbolic discounting by allowing the effective discount factor to increase over the first two consecutive trade-offs. Formally, these preferences specify an effective discount factor of $\beta \delta \gamma$ for the first adjacent trade-off, and an effective discount factor of $\gamma \delta$ for the second adjacent trade-off. For any other two consecutive future periods beyond that, the effective discount factor is assumed to be $\delta$. If $\beta \in(0,1)$ and $\gamma \in(0,1)$, the effective discount factor would be increasing for two consecutive trade-offs (rather than just one as with order-one discounting).

We construct the order-two quasi-hyperbolic discount function as follows. By assumption $D_{2}(0)=1$. Since the effective discount factor for the trade-off between the current period and 
the next is $\beta \delta \gamma$, we must have $D_{2}(1)=\beta \delta \gamma D_{2}(0)=\beta \delta \gamma$. Similarly, the discount factor for the trade-off between the next period and the one thereafter must be $\gamma \delta$, implying that $D_{2}(2)=$ $\gamma \delta D_{2}(1)=\beta \delta^{2} \gamma^{2}$. Finally, for $\forall \tau \geqslant 3$, we have $D_{2}(\tau)=\delta D_{2}(\tau-1)$, so $D_{2}(\tau)=\beta \gamma^{2} \delta^{\tau}$. To recap, our order-two specification is defined as

$$
D_{2}(0)=1, \quad D_{2}(1)=\beta \delta \gamma, \quad D_{2}(\tau)=\beta \gamma^{2} \delta^{\tau}, \forall \tau \geqslant 2
$$

Note that order-one quasi-hyperbolic discounting is a special case of this specification which occurs when $\gamma=1$.

\subsection{Recursive representation of payoffs}

Now we formulate the lifetime payoff of an agent with order-two quasi-hyperbolic preferences recursively. This formulation will be used later when applying dynamic programming techniques to study the intra-personal game of decision makers with such preferences.

The lifetime payoff of the period- $t$ agent is defined as

$$
U^{t}=u^{t}+\beta \delta \gamma u^{t+1}+\beta \gamma^{2} \delta^{2} u^{t+2}+\beta \gamma^{2} \delta^{3} u^{t+3}+\beta \gamma^{2} \delta^{4} u^{t+4}+\ldots
$$

Let $V^{t+1}$ be the present value of the payoffs that are expected to accrue after today:

$$
V^{t+1}=u^{t+1}+\gamma \delta u^{t+2}+\gamma \delta^{2} u^{t+3}+\gamma \delta^{3} u^{t+4}+\ldots
$$

With this notation, (10) can be rewritten as:

$$
U^{t}=u^{t}+\beta \delta \gamma V^{t+1}
$$


Moreover, note that $V^{t+1}$ would be the lifetime payoff of an agent with order-one quasi-hyperbolic preferences. In Section 2.3, we showed that it can be represented as

$$
V^{t+1}=u^{t+1}+\gamma \delta W^{t+2},
$$

where $W^{t+2}$ is the lifetime payoff of a period- $t+2$ agent with exponential preferences:

$$
W^{t+2}=u^{t+2}+\delta W^{t+3}
$$

To recap, the lifetime payoff of a period- $t$ agent with order-two quasi-hyperbolic preferences can be written as

$$
U^{t}=u^{t}+\beta \delta \gamma V^{t+1}
$$

where $V^{t+1}$ is defined as

$$
V^{t+1}=u^{t+1}+\gamma \delta W^{t+2},
$$

and $W^{t+2}$ satisfies

$$
W^{t+2}=u^{t+2}+\delta W^{t+3} \text {. }
$$

\section{Order-three quasi-hyperbolic discounting}

Next we study an alternative generalisation of $(\beta, \delta)$ discounting. We will refer to it as "order-three quasi-hyperbolic discounting". This specification also gives rise to lifetime payoffs that are amenable to recursive representation. Unlike the previous generalisation, however, it exhibits decreasing impatience over the entire planning horizon. That is, $D_{3}\left(\tau^{\prime}+1\right) / D_{3}\left(\tau^{\prime}\right)>D_{3}\left(\tau^{\prime \prime}+1\right) / D_{3}\left(\tau^{\prime \prime}\right)$ for all $\tau^{\prime}>\tau^{\prime \prime}$. We will show that this feature makes order-three quasi-hyperbolic discounting a very good approximation to the true hyperbolic discount function. As with the previous generalisation, 
these preferences also nest order-one quasi-hyperbolic discounting as a special case. Finally, the added analytical complexity relative to order-one quasi-hyperbolic discounting is relatively minor.

\subsection{Recursive definition of the discount function}

We consider the following recursive definition of the order-three quasi-hyperbolic discount function:

$$
D_{3}(\tau+1)=\beta \delta^{\tau+1}+\gamma D_{3}(\tau)
$$

where $D_{3}(0)=1$, and $\beta, \delta, \gamma \in(0,1)$. Note that this specification constitutes a generalisation of order-one quasi-hyperbolic discounting. In particular, $(\beta, \delta)$ preferences are a special case of (16) which arises when $\gamma=0$. Since the properties of this special case are well-known, we now focus on settings where $\gamma>0$. We will show that these preferences may give rise to an increasing effective discount factor $D_{3}(\tau+1) / D_{3}(\tau)$ for all $\tau$. This feature will allow us to attain a better approximation to true hyperbolic discounting.

\subsection{Closed-form representation}

We can express (16) as a function of time only. In particular, it can be shown that (16) is equivalent to

$$
D_{3}(\tau)=\left(\frac{\beta \delta^{\tau+1}-\gamma^{\tau+1}+\delta(1-\beta) \gamma^{\tau}}{\delta-\gamma}\right), \tau \geqslant 1
$$

Below we present two alternative proofs.

\subsubsection{Proof via induction}

To prove the above result, we could use induction. First we verify that setting $\tau=1$ and $\tau=2$ in (17) would give us expressions identical to those generated by (16). Indeed, evaluating (17) at 
$\tau=1$ and $\tau=2$ yields

$$
\begin{aligned}
& D_{3}(1)=\left(\frac{\beta \delta^{2}-\gamma^{2}+\delta(1-\beta) \gamma}{\delta-\gamma}\right)=\beta \delta+\gamma \\
& D_{3}(2)=\left(\frac{\beta \delta^{3}-\gamma^{3}+\delta(1-\beta) \gamma^{2}}{\delta-\gamma}\right)=\beta \delta^{2}+\gamma(\beta \delta+\gamma)
\end{aligned}
$$

Then we assume that (17) holds for an arbitrary $\tau$. We will show that it will also hold for $\tau+1$.

Substituting (17) in the right-hand side of (16) delivers

$$
\beta \delta^{\tau+1}+\gamma\left(\frac{\beta \delta^{\tau+1}-\gamma^{\tau+1}+\delta(1-\beta) \gamma^{\tau}}{\delta-\gamma}\right)
$$

Simplifying (18) yields

$$
\left(\frac{\beta \delta^{\tau+2}-\gamma^{\tau+2}+\delta(1-\beta) \gamma^{\tau+1}}{\delta-\gamma}\right)
$$

which is identical to (17) evaluated at $\tau+1$. This completes our induction proof.

\subsubsection{Proof by solving a homogeneous difference equation}

Alternatively, we can obtain the discount function by solving a difference equation. Note that

$$
D_{3}(\tau+2)-\gamma D_{3}(\tau+1)=\beta \delta^{\tau+2}=\delta\left(D_{3}(\tau+1)-\gamma D_{3}(\tau)\right) .
$$

Rearranging gives us

$$
D_{3}(\tau+2)-(\gamma+\delta) D_{3}(\tau+1)+\gamma \delta D_{3}(\tau)=0
$$

Condition (19) is a homogeneous difference equation of order two with $D_{3}(1)=\beta \delta+\gamma$ and $D_{3}(2)=$

$\beta \delta^{2}+\gamma(\beta \delta+\gamma)$. This equation can be solved using standard techniques. Let $\chi_{1}$ and $\chi_{2}$ be the 
roots to the characteristic equation

$$
\chi^{2}-(\gamma+\delta) \chi+\gamma \delta=0
$$

That is,

$$
\chi_{1}=\delta, \quad \chi_{2}=\gamma .
$$

The solution to (19) is given by

$$
D_{3}(\tau)=c_{1} \delta^{\tau}+c_{2} \gamma^{\tau},
$$

where the constants $c_{1}, c_{2}$ are chosen to match $D_{3}(1)$ and $D_{3}(2)$ :

$$
\begin{aligned}
c_{1} \delta+c_{2} \gamma & =\beta \delta+\gamma \\
c_{1} \delta^{2}+c_{2} \gamma^{2} & =\beta \delta^{2}+\gamma(\beta \delta+\gamma) .
\end{aligned}
$$

Solving this system of equations for $c_{1}$ and $c_{2}$ yields

$$
c_{1}=\frac{\delta \beta}{(\delta-\gamma)}, \quad c_{2}=\frac{(1-\beta) \delta-\gamma}{(\delta-\gamma)}
$$

Substituting $c_{1}, c_{2}$ in (20) and rearranging delivers (17).

\subsection{Gradually increasing discount factor}

Expression (17) is helpful because we can use it to establish the range of parameter values for which the effective discount factor $D_{3}(\tau+1) / D_{3}(\tau)$ will increasing for all $\tau$. As discussed earlier, the orderone and order-two approximations lack this property. In particular, they specify constant discount factors for trade-offs beyond a given period: $D_{1}(\tau+1) / D_{1}(\tau)=\delta, \forall \tau \geqslant 1$, and $D_{2}(\tau+1) / D_{2}(\tau)=$ 
$\delta, \forall \tau \geqslant 2$.

We use (17) to obtain a closed-form expression for $D_{3}(\tau+1) / D_{3}(\tau)$ :

$$
\frac{D_{3}(\tau+1)}{D_{3}(\tau)}=\frac{\beta \delta^{\tau+2}-\gamma^{\tau+2}+\delta(1-\beta) \gamma^{\tau+1}}{\beta \delta^{\tau+1}-\gamma^{\tau+1}+\delta(1-\beta) \gamma^{\tau}}
$$

Simplifying the above expression yields

$$
\frac{D_{3}(\tau+1)}{D_{3}(\tau)}=\frac{\beta \delta-\gamma\left(\frac{\gamma}{\delta}\right)^{\tau+1}+\delta(1-\beta)\left(\frac{\gamma}{\delta}\right)^{\tau+1}}{\beta-\left(\frac{\gamma}{\delta}\right)^{\tau+1}+\delta \frac{(1-\beta)}{\gamma}\left(\frac{\gamma}{\delta}\right)^{\tau+1}}=\frac{\gamma \beta(\delta-\gamma)}{\gamma \beta+[\delta(1-\beta)-\gamma]\left(\frac{\gamma}{\delta}\right)^{\tau+1}}+\gamma
$$

From (21) we can infer that the agent's effective discount factor will be gradually increasing in $\tau$ if

$$
\delta(1-\beta)>\gamma
$$

When $\beta \in(0,1)$, this condition ensures that $\delta>\gamma$. Thus, as $\tau$ keeps increasing, the denominator of the right-hand side of (21) will gradually become smaller, whilst the numerator $\gamma \beta(\delta-\gamma)$ will remain unchanged. To satisfy (22), the parameter $\gamma$ should be sufficiently close to 0 . Moreover, this condition will never hold for any positive $\gamma$ if $\beta=1$.

Note that, as $\tau \longrightarrow \infty$, the effective discount factor between two adjacent periods (21) converges to $\delta$. Thus, order-three quasi-hyperbolic discounting implies a long-term (asymptotic) discount factor of $\delta$. This observation highlights an important difference between our specification and true hyperbolic discounting: $D_{h}(\tau+1) / D_{h}(\tau)$ converges to 1 instead.

We can rewrite $(22)$ as $\beta \delta+\gamma<\delta$. The interpretation of this condition is that the effective discount factor will be gradually increasing as long as $D_{3}(1) / D_{3}(0)$ is less than the long-term discount factor. 


\subsection{Recursive representation of payoffs}

The recursive definition (16) of the order-three quasi-hyperbolic discount function can be used to formulate the decision maker's lifetime payoff recursively. From the viewpoint of period $t$, this payoff is given by

$$
U^{t}=\sum_{\tau=0}^{\infty} D_{3}(\tau) u^{t+\tau} .
$$

When period $t+1$ arrives, the lifetime payoff from that perspective will be:

$$
U^{t+1}=\sum_{\tau=0}^{\infty} D_{3}(\tau) u^{t+\tau+1}
$$

We would like to obtain an equation which describes the agent's lifetime payoff through itself. Multiplying (24) by $\gamma$ and subtracting it from (23) yields

$$
U^{t}-\gamma U^{t+1}=u^{t}+\sum_{\tau=1}^{\infty}\left[D_{3}(\tau)-\gamma D_{3}(\tau-1)\right] u^{t+\tau}
$$

However, the recursive definition (16) of the discount function suggests that $D_{3}(\tau)-\gamma D_{3}(\tau-1)=$ $\beta \delta^{\tau-1}, \forall \tau$. Hence, we can rewrite equation (25) as:

$$
U^{t}-\gamma U^{t+1}=u^{t}+\beta \sum_{\tau=1}^{\infty} \delta^{\tau} u^{t+\tau} .
$$

Note that the right-hand side of $(26)$ is simply the period- $t$ lifetime payoff of an agent with $(\beta, \delta)$ preferences. Let

$$
V^{t}=u^{t}+\beta \sum_{\tau=1}^{\infty} \delta^{\tau} u^{t+\tau}
$$

Using Laibson's method described in section 2.3.2, we can formulate $V^{t}$ recursively. Define the 
following sum:

$$
W^{t+1}=\sum_{\tau=1}^{\infty} \delta^{\tau-1} u^{t+\tau} .
$$

Thus, we can express $V^{t}$ as

$$
V^{t}=u^{t}+\beta \delta W^{t+1}
$$

As noted earlier, from the viewpoint of a period- $t$ agent with $(\beta, \delta)$ preferences, discounting becomes exponential from $t+1$ onward. Hence,

$$
W^{t+1}=u^{t+1}+\delta W^{t+2} .
$$

To recap, the lifetime payoff $U^{t}$ of an agent with order-three quasi-hyperbolic preferences can be represented recursively as follows:

$$
U^{t}=u^{t}+\gamma U^{t+1}+\beta \delta W^{t+1},
$$

where $W^{t+1}$ satisfies

$$
W^{t+1}=u^{t+1}+\delta W^{t+2} .
$$

Note that if $\gamma=0$, the above recursive formulation reduces to that for $(\beta, \delta)$ preferences described in Section 2.3.2. Finally, using (8), we could rewrite (26) in the following form:

$$
U^{t}=(1-\beta) u^{t}+\gamma U^{t+1}+\beta W^{t} .
$$




\section{Comparison and calibration}

Now we argue that $D_{2}(\tau)$ and, even more so, $D_{3}(\tau)$ can be calibrated to deliver a better approximation to true hyperbolic discounting relative to $D_{1}(\tau)$. We constructed our order-two and order-three approximations to nest $(\beta, \delta)$ preferences as a special case. Thus, for appropriately chosen values of $\gamma$, both our generalisations are guaranteed to deliver a closer fit to the true hyperbolic discount function. The question of interest is whether this improvement is significant for plausible parameter values of $(2)$.

To answer this question, we will use a numerical approach. Specifically, we calibrate the various quasi-hyperbolic discount functions to match $D_{h}(\tau)$ for a range of values of the parameter $d$. Let $L_{1}(\beta, \delta, \gamma), L_{2}(\beta, \delta, \gamma)$ and $L_{3}(\beta, \delta, \gamma)$ be the sums of squared differences between the relevant discount function and the true hyperbolic discount function $D_{h}(\tau)$ over the first $T$ periods:

$$
L_{i}(\beta, \delta, \gamma)=\sum_{\tau=1}^{T}\left(D_{i}(\tau)-D_{h}(\tau)\right)^{2}, \quad i=1,2,3,
$$

We look for the values of the preference parameters $\beta, \delta, \gamma$ that minimise these expressions.

Our method is as follows. First we assign a particular value to the parameter $d$ in $D_{h}(t)$. Then we compute the values $\beta^{*}, \delta^{*}, \gamma^{*}$ which minimise $L_{i}(\beta, \delta, \gamma) .{ }^{4}$ As a measure of the goodness of fit we could use the value of the minimised sum of squared differences $L_{i}^{*}=L_{i}\left(\beta^{*}, \delta^{*}, \gamma^{*}\right)$. The parameter $T$ is set at $T=15$. The calibrated preference parameters are reported in Table 1 .

First, note that when $d$ is low, our order-two approximation requires a value of $\beta$ above 1 in order to best fit true hyperbolic discounting. If $\beta>1$, however, the effective discount factor is not monotonically increasing in time. Since this contradicts the stylised fact about decreasing impatience, we should either avoid this approximation in such cases, or use an alternative method

\footnotetext{
${ }^{4}$ The parameter $\gamma$ is absent from order-one quasi-hyperbolic discounting, and so will not be reported.
} 
for calibration.

The table demonstrates that both $D_{2}(\tau)$ and $D_{3}(\tau)$ deliver a better fit to true hyperbolic discounting relative to $D_{1}(\tau)$. The improvement in precision when using our order-three specification is bigger: the ratio $L_{2}^{*} / L_{1}^{*}$ is between .5443 and .3668 , while the ratio $L_{3}^{*} / L_{1}^{*}$ is in the range .0168 to .0701. The intuition for this result is straightforward. As order-three quasi-hyperbolic discounting delivers a gradually increasing discount factor, this specification is able to approximate true hyperbolic discounting more closely. Furthermore, note that the relative precision of the order-two approximation improves for higher values of $d$, while that of the order-three approximation is better for lower values of $d$.

The goodness of fit of our generalisations to true hyperbolic discounting can also be illustrated graphically. Figure 1a and Figure 1b plot the calibrated values of the order-two and order-three quasi-hyperbolic discount functions, as well as the corresponding values of the true hyperbolic discount function, for $d=0.7$. As Figure $1 \mathrm{~b}$ demonstrates, the order-three approximation in particular matches true hyperbolic discounting very closely.

To emphasise this point, Figure $2 \mathrm{a}$ and Figure $2 \mathrm{~b}$ plot the effective discount factors for the two generalisations, $D_{2}(\tau+1) / D_{2}(\tau)$ and $D_{3}(\tau+1) / D_{3}(\tau)$, next to the effective discount factors $D_{h}(\tau+$ 1) $/ D_{h}(\tau)$ of the true hyperbolic discount function with $d=0.7$. As our order-three specification yields a gradually increasing effective discount factor, it manages to deliver a much better fit.

An alternative approach to calibration would be to minimise the weighted sum of squared differences between the values of the true hyperbolic discount function and the relevant approximation. It can be argued that periods that are closer to the agent's perspective are more important for him, so they should have higher weights. A natural candidate for a set of weights would be given by the values of the true hyperbolic discount function. Thus, we could also choose the preference 
parameters to minimise the following objective functions:

$$
\tilde{L}_{i}(\beta, \delta, \gamma)=\sum_{\tau=1}^{T} D_{h}(\tau)\left(D_{i}(\tau)-D_{h}(\tau)\right)^{2}, \quad i=1,2,3
$$

The values of $\beta, \delta, \gamma$ calibrated in this way are shown in Table 2 .

\section{General intra-personal game}

The previous section demonstrated that our generalised discount functions are able to produce values that are much closer to true hyperbolic discounting relative to Laibson's approach. Next we study the implications of this better fit for the predictions of economic models. In Section 7 we will consider two popular economic applications of quasi-hyperbolic discounting. In these special cases, we can directly compute the Markov-perfect equilibrium (MPE) for true hyperbolic discounters. We compare these equilibria to the predictions of the models with calibrated quasi-hyperbolic discounting of order one, two and three. The comparison demonstrates that our order-two, and especially order-three, approximations yield policies and strategies that are very close to those generated by true hyperbolic discounting.

To do this comparison, we now analyse a general setting in which a stock variable evolves according to a linear law of motion. The two economic applications we will explore in Section 7 are special cases of this setting. Using the recursive representations of lifetime payoffs derived earlier, we formulate Bellman equations for decision makers with order one, two and three quasi-hyperbolic discount functions. Dynamic programming techniques enable us to derive Euler equations that characterise their MPE strategies. We also provide an equilibrium condition for true hyperbolic agents with a special class of utility functions. 


\subsection{General setting}

Suppose that the decision maker's period-t utility is given by $u\left(x^{t}, k^{t}\right)$, where $x^{t}$ is his current consumption and $k^{t}$ is a stock variable. The utility is strictly increasing in $x^{t}$ and satisfies the Inada conditions. It may or may not depend directly on $k^{t}$. The stock evolves according to the following law of motion:

$$
k^{t+1}=a k^{t}+b x^{t}
$$

In the next section, we will analyse a couple of special cases of this general setting, where the variables and the parameters will have specific economic interpretations.

As Strotz (1955-1956) demonstrated, unless decision makers have exponential preferences, they will have a time consistency problem. There are several ways of modelling the behaviour of such decision makers. In this paper, we assume that they are sophisticated. That is, they are aware of their time consistency problem and take mitigating actions. We will model their choices as an intra-personal dynamic game. Our focus will be on the MPE of this game. In other words, we consider a consumption strategy that is a differentiable function of the current state: $x^{t}=h\left(k^{t}\right)$. Note that such an equilibrium is subgame perfect: a player's strategy will be optimal for any value of $k^{t}$, and thus for any history.

\subsection{Exponential discounting}

First, we study the benchmark case of exponential discounting. Suppose that the agent's discount function is given by (1). As discussed earlier, these preferences would give rise to an optimal consumption plan that is time consistent. To characterise it, we use dynamic programming. Let the agent's value function be $W\left(k^{t}\right)$. His Bellman equation is

$$
W\left(k^{t}\right)=\max _{x^{t}}\left\{u\left(x^{t}, k^{t}\right)+\delta W\left(a k^{t}+b x^{t}\right)\right\} .
$$


Differentiating the right-hand side of (30) with respect to $x^{t}$ yields the first-order condition

$$
u_{x}^{t}+\delta b W_{k}^{t+1}=0
$$

Thus, $W_{k}^{t+1}=-u_{x}^{t} /(\delta b)$. Furthermore, differentiating (30) with respect to $k^{t}$ gives us an envelope condition:

$$
W_{k}^{t}=u_{k}^{t}+\delta a W_{k}^{t+1}
$$

Substituting the expressions for $W_{k}^{t}$ and $W_{k}^{t+1}$ in this envelope condition delivers the agent's Euler equation:

$$
u_{x}^{t}+\delta b u_{k}^{t+1}-\delta a u_{x}^{t+1}=0 .
$$

Essentially condition (31) is a difference-differential equation. For some specifications of the utility function $u$, it can be used to obtain exact solutions for the optimal feedback rule $h\left(k^{t}\right)$. In later sections, we will compute such solutions.

\subsection{Order-one quasi-hyperbolic discounting}

Now we assume that the consumer has order-one quasi-hyperbolic preferences. That is, his discount function is defined by Laibson's specification (3). These preferences would give rise to a time consistency problem for the decision maker. To characterise his behaviour, we model consumption choices as an intra-personal game between his current and the future selves. As explained earlier, we focus on the Markov-perfect equilibrium of this game. Let the agent's MPE strategy be $x^{t}=$ $h\left(k^{t}\right), \forall t$.

We will use a method proposed by Laibson to derive a condition characterising this strategy. The recursive formulation (5), (6) in Section 3 suggests that equilibrium consumption will solve 
the Bellman equation

$$
V\left(k^{t}\right)=\max _{x^{t}}\left\{u\left(x^{t}, k^{t}\right)+\delta \beta W\left(a k^{t}+b x^{t}\right)\right\}
$$

where the continuation value function $W$ satisfies

$$
W\left(k^{t}\right)=u\left(h\left(k^{t}\right), k^{t}\right)+\delta W\left(a k^{t}+b h\left(k^{t}\right)\right) .
$$

Differentiating the right-hand side of (32) with respect to $x^{t}$ yields the first-order condition

$$
u_{x}^{t}-\beta \delta b W_{k}^{t+1}=0 .
$$

Thus, $W_{k}^{t+1}=u_{x}^{t} /(b \beta \delta)$. Moreover, differentiating (33) with respect to $k^{t}$ gives us

$$
W_{k}^{t}=h_{k}^{t} u_{x}^{t+1}+u_{k}^{t+1}+\delta\left(a+b h_{k}^{t}\right) W_{k}^{t+1}
$$

We substitute $W_{k}^{t}$ and $W_{k}^{t+1}$ in the above condition to obtain the agent's generalised Euler equation:

$$
u_{x}^{t}+b \beta \delta u_{k}^{t+1}-\delta\left[a+b(1-\beta) h_{k}^{t+1}\right] u_{x}^{t+1}=0 .
$$

A comparison with (31) shows that now the equilibrium condition contains the extra term $\delta b(1-$ $\beta) h_{k}^{t+1} u_{x}^{t+1}$. This term accounts for the strategic considerations in the intra-personal game between the current consumer and his future self. Note that when $\beta=1$ the intra-personal strategic effect disappears and (34) reduces to (31). 


\subsection{Order-two quasi-hyperbolic discounting}

Next we consider the first of our two generalisations. Suppose that the agent has order-two quasihyperbolic preferences as defined by (9). Just as in the setting with order-one quasi-hyperbolic discounting, the consumer will have a time consistency problem. Again, we focus on the Markovperfect equilibrium of his intra-personal game. Let the MPE strategy be $x^{t}=h\left(k^{t}\right)$. To characterise $h$, we will apply dynamic programming techniques to the recursive formulation (13), (14), (15) derived in Section 4. The agent's Bellman equation is now given by:

$$
U\left(k^{t}\right)=\max _{x^{t}}\left\{u\left(x^{t}, k^{t}\right)+\beta \gamma \delta V\left(a k^{t}+b x^{t}\right)\right\}
$$

where $V$ is defined by

$$
V\left(k^{t}\right)=u\left(h\left(k^{t}\right), k^{t}\right)+\gamma \delta W\left(a k^{t}+b h\left(k^{t}\right)\right) .
$$

In addition, (15) suggests that the third value function, $W$, satisfies the functional equation

$$
W\left(k^{t}\right)=u\left(h\left(k^{t}\right), k^{t}\right)+\delta W\left(a k^{t}+b h\left(k^{t}\right)\right) .
$$

Taking the first-order condition of (35), we get:

$$
u_{x}^{t}=\beta \gamma \delta b V_{k}^{t+1}
$$

Differentiating (36) and (37) with respect to $k^{t}$ gives us

$$
V_{k}^{t}=h_{k}^{t} u_{x}^{t}+u_{k}^{t}+\gamma \delta\left(a+b h_{k}^{t}\right) W_{k}^{t+1}
$$


and

$$
W_{k}^{t}=h_{k}^{t} u_{x}^{t}+u_{k}^{t}+\delta\left(a+b h_{k}^{t}\right) W_{k}^{t+1}
$$

respectively. From (38), we obtain

$$
V_{k}^{t+1}=-\frac{u_{x}^{t}}{\beta \gamma \delta b}
$$

Substituting $V_{k}^{t}$ into (39) yields

$$
W_{k}^{t+1}=-\frac{1}{\gamma \delta\left(a+b h_{k}^{t}\right)}\left[\frac{u_{x}^{t-1}}{\beta \gamma \delta b}+h_{k}^{t} u_{x}^{t}+u_{k}^{t}\right]
$$

Plugging $W_{k}^{t}$ and $W_{k}^{t+1}$ into (40) delivers the following equation:

$u_{x}^{t}+\left(h_{k}^{t+1} u_{x}^{t+1}+u_{k}^{t+1}\right) \beta \gamma \delta b+\left[1-\frac{1}{\gamma}\right] \gamma \delta\left(a+b h_{k}^{t+1}\right) \beta \gamma \delta b\left(h_{k}^{t+2} u_{x}^{t+2}+u_{k}^{t+2}\right)-\gamma \delta\left(a+b h_{k}^{t+1}\right) u_{x}^{t+1}=0$

Simplifying this equation yields a necessary condition for the decision maker's MPE strategy:

$$
u_{x}^{t}+\beta \gamma \delta b\left(h_{k}^{t+1} u_{x}^{t+1}+u_{k}^{t+1}\right)-\delta\left(a+b h_{k}^{t+1}\right)\left[u_{x}^{t+1}+\delta \beta b \gamma(1-\gamma)\left(h_{k}^{t+2} u_{x}^{t+2}+u_{k}^{t+2}\right)\right]=0
$$

In the special case when $\gamma=1$, this condition reduces to the order-one quasi-hyperbolic Euler equation (34).

\subsection{Order-three quasi-hyperbolic discounting}

Similarly, consider a consumer whose discount function is order-three quasi-hyperbolic, i.e. defined by (16). The recursive representation of the lifetime payoff of an agent with such preferences was given by (27), (28). Hence, his optimal consumption strategy would solve the following Bellman 
equation:

$$
U\left(k^{t}\right)=\max _{x^{t}}\left\{u\left(x^{t}, k^{t}\right)+\gamma U\left(a k^{t}+b x^{t}\right)+\delta \beta W\left(a k^{t}+b x^{t}\right)\right\},
$$

where $W$ satisfies the functional equation

$$
W\left(k^{t}\right)=u\left(h\left(k^{t}\right), k^{t}\right)+\delta W\left(a k^{t}+b h\left(k^{t}\right)\right) .
$$

We derive this agent's generalised Euler equation using the same techniques as before. First, differentiating the right-hand side of (42) yields the first-order condition

$$
u_{x}^{t}+\gamma b U_{k}^{t+1}+\beta \delta b W_{k}^{t+1}=0 .
$$

Also, differentiating (42) with respect to $k^{t}$ gives us an envelope condition,

$$
U_{k}^{t}=u_{k}^{t}+\gamma a U_{k}^{t+1}+\beta \delta a W_{k}^{t+1}
$$

Equations (44) and (45) together imply that

$$
U_{k}^{t}=u_{k}^{t}-a u_{x}^{t} / b
$$

Substituting $U_{k}^{t}$ in (44) delivers an expression for $W_{k}^{t+1}$ :

$$
W_{k}^{t+1}=-\frac{u_{x}^{t}+\gamma\left(b u_{k}^{t+1}-a u_{x}^{t+1}\right)}{\beta \delta b} .
$$

Finally, differentiating (43) with respect to $k^{t}$ yields

$$
W_{k}^{t}=h_{k}^{t} u_{x}^{t}+u_{k}^{t}+\delta\left(a+b h_{k}^{t}\right) W_{k}^{t+1} .
$$


If we substitute $W_{k}^{t}$ and $W_{k}^{t+1}$ in that condition, we obtain the generalised Euler equation

$$
u_{x}^{t}+b \beta \delta u_{k}^{t+1}-\delta\left[a+b(1-\beta) h_{k}^{t+1}\right] u_{x}^{t+1}-\gamma\left[a u_{x}^{t+1}-b u_{k}^{t+1}-\delta\left(a+b h_{k}^{t+1}\right)\left(a u_{x}^{t+2}-b u_{k}^{t+2}\right)\right]=0 .
$$

Note that if $\gamma=0,(46)$ becomes identical to the Euler equation (34) for $(\beta, \delta)$ preferences.

\subsection{True hyperbolic discounting with homogeneous payoffs}

Although a general solution to the above intra-personal game with true hyperbolic discounting is not available, Calcott and Petkov (2015) managed to characterise its Markov-perfect equilibrium for a special class of payoff functions. In particular, they focus on payoffs that are homogeneous of some degree $\eta$. That is, $u(x, k)$ satisfies

$$
u(s x, s k)=s^{\eta} u(x, k), \quad \forall s>0 .
$$

Such a specification yields an MPE strategy that is proportional to the state variable: $x^{t}=\lambda k^{t}$. If the law of motion of $k^{t}$ is as given by (29), on the equilibrium path instantaneous payoffs will exponentially converge to 0 . In particular, if agents follow their equilibrium strategies, we will have $u^{t}=\left(k^{1}\right)^{\eta(t-1)}(a+b \lambda)^{\eta(t-1)} u(\lambda, 1)$. This suggests that the agent's lifetime payoff may be finite.

As already argued, this problem cannot be formulated recursively. However, we could characterise the MPE strategy using the one-shot deviation principle. Suppose that all agents from period $t+1$ onward will follow the equilibrium strategy $x=\lambda k$, and consider the problem of of the decision maker in period $t$. He chooses $x^{t}$ to maximise his lifetime payoff $U^{t}=\sum_{\tau=0}^{\infty} D_{h}(\tau) u\left(x^{t+\tau}, k^{t+\tau}\right)$, where $x^{t+\tau}=\lambda k^{t+\tau}$ for $\tau \geqslant 1$. The homogeneity assumption implies that $U^{t}$ can be rewritten as

$$
U^{t}=u\left(x^{t}, k^{t}\right)+\left(b x^{t}+a k^{t}\right)^{\eta} u(\lambda, 1) \sum_{\tau=1}^{\infty} D_{h}(\tau)(b \lambda+a)^{\eta(\tau-1)} .
$$


Note that $U^{t}$ will be finite only if

$$
(b \lambda+a)^{\eta}<1
$$

Differentiating (47) with respect to $x^{t}$ yields the first-order condition

$$
u_{x}\left(x^{t}, k^{t}\right)+b \eta\left(b x^{t}+a k^{t}\right)^{\eta-1} u(\lambda, 1) \sum_{\tau=1}^{\infty} D_{h}(\tau)(b \lambda+a)^{\eta(\tau-1)}=0
$$

The MPE strategy must be time invariant. Hence, the optimal current consumption must be $\lambda k^{t}$. Also, $u_{x}$ is homogeneous of degree $\eta-1$. Thus, we can factor out $\left(k^{t}\right)^{\eta-1}$.

$$
u_{x}(\lambda, 1)+b \eta(b \lambda+a)^{\eta-1} u(\lambda, 1) \sum_{\tau=1}^{\infty} D_{h}(\tau)(b \lambda+a)^{\eta(\tau-1)}=0
$$

Substituting $D_{h}(\tau)$ from (2) and rearranging yields

$$
u_{x}(\lambda, 1)+\frac{b \eta}{(b \lambda+a)} u(\lambda, 1) \frac{1}{d} \sum_{\tau=1}^{\infty} \frac{(b \lambda+a)^{\eta \tau}}{(1 / d+\tau)}=0
$$

Finally, we can rewrite the above condition as

$$
u_{x}(\lambda, 1)+\frac{b \eta}{(b \lambda+a)} u(\lambda, 1) \frac{1}{d}\left[\Phi\left((b \lambda+a)^{\eta}, 1,1 / d\right)-d\right]=0 .
$$

The function $\Phi$ is known as the Lerch transcendent:

$$
\Phi(z, \zeta, v)=\sum_{\tau=0}^{\infty} \frac{z^{\tau}}{(v+\tau)^{\zeta}}
$$

This function is well-defined for $|z|<1$ and its values can be computed with standard mathematical software such as Maple or Mathematica. ${ }^{5}$

\footnotetext{
${ }^{5}$ An alternative approach is to consider a finite horizon setting with true hypebolic consumer. Such a game can be
} 
To recap, the parameter $\lambda$ of the MPE strategy of a true hyperbolic consumer with instantaneous utility that is homogeneous of degree $\eta$ solves the necessary condition

$$
u_{x}(\lambda, 1)+\frac{b \eta u(\lambda, 1)}{(b \lambda+a)} \phi(\lambda)=0
$$

where

$$
\phi(\lambda)=\frac{\Phi\left((b \lambda+a)^{\eta}, 1,1 / d\right)}{d}-1
$$

It should be pointed out that (51) may not have an interior solution for all admissible utility functions. For example, in consumption - saving models, the agent may be best off postponing all of his consumption indefinitely, i.e. choosing $x^{t}=0$ in all periods.

\section{Economic Applications}

Now we apply the analysis of the general setting from the previous section to two specific economic problems. These problems are well-known in the literature. They concern phenomena that would be difficult to explain within a neoclassical economic framework. In fact, they motivated much of the existing research on hyperbolic discounting and its behavioural implications.

These two applications constitute special cases of the general setting studied above. The analysis from Section 6 enables us to compute the equilibria for agents with true hyperbolic discounting, and compare them to the predictions of the models based on the various quasi-hyperbolic approximations. Our numerical examples demonstrate the order-three discounting, and to a lesser extent order-two discounting, yield policies that are much closer to those of true hyperbolic discounters as compared to Laibson's approach.

solved numerically using backward induction. As the time horizon is extended, equilibrium strategy should converge to that of the infinite-horizon game. 


\subsection{Application one: consumption - saving model}

Quasi-hyperbolic discounting was given particular prominence by Laibson (1998). In that paper, $(\beta, \delta)$ preferences were used to explain the low saving rate in the United States. Now we investigate how our generalisations affect the results of this model.

\subsubsection{Setting}

To illustrate the underlying mechanism, we will use a simple buffer stock model. Consider an agent who has a capital stock $k^{t}$. He chooses his current consumption $x^{t}$, and attains an instantaneous utility $u\left(x^{t}\right)$. Initially we are agnostic about the functional form of $u$. The only restriction is that it does not depend on $k^{t}$ directly: $u_{k}^{t} \equiv 0$. Later, when computing specific examples, we will assume constant relative risk aversion utility: $u(x)=\left(x^{1-\sigma}-1\right) /(1-\sigma)$. In the special case when $\sigma \rightarrow 1$, this utility function converges to $u(x)=\ln (x)$.

The agent saves the capital that is not used for consumption. Suppose that, in each period, the gross interest rate is $R$. Thus, the law of motion of $k^{t}$ is given by (29), where $a=R$ and $b=-R$. The agent's objective is to maximise his lifetime utility subject to the above law of motion of capital.

\subsubsection{Consumption strategies}

Next we characterise the decision maker's equilibrium consumption strategies.

Exponential discounting First, suppose that the decision maker's discount function is exponential with factor $\delta$. When $a=R, b=-R$ and $u_{k}^{t} \equiv 0$, Euler equation (31) takes the following form:

$$
u_{x}^{t}-\delta R u_{x}^{t+1}=0
$$


Consider the example with $u(x)=\left(x^{1-\sigma}-1\right) /(1-\sigma)$. We conjecture that the optimal feedback rule is given by $x^{t}=\lambda_{0} k^{t}$. Substitution in the Euler equation gives us a condition for $\lambda_{0}$ :

$$
1-\frac{\delta R^{1-\sigma}}{\left(1-\lambda_{0}\right)^{\sigma}}=0
$$

In the limit case when $u(x)=\ln (x)$, we have

$$
\lambda_{0}=(1-\delta)
$$

Order-one quasi-hyperbolic discounting Next, suppose that the consumer is an order-one quasi-hyperbolic discounter. Assigning $a=R, b=-R$ and $u_{k}^{t} \equiv 0$ to Euler equation (34), we get

$$
u_{x}^{t}-\delta R\left[1-(1-\beta) h_{k}^{t+1}\right] u_{x}^{t+1}=0 .
$$

In our example with $u(x)=\left(x^{1-\sigma}-1\right) /(1-\sigma)$, we conjecture an MPE strategy with form $x^{t}=\lambda_{1} k^{t}$. Substitution in Euler equation (34) delivers the following condition for $\lambda_{1}$ :

$$
1-\frac{\delta R^{1-\sigma}\left[1-\lambda_{1}(1-\beta)\right]}{\left(1-\lambda_{1}\right)^{\sigma}}=0
$$

In the limit case when $u(x)=\ln (x)$, solving for $\lambda_{1}$ yields

$$
\lambda_{1}=\frac{1-\delta}{1-\delta(1-\beta)}
$$


Order-two quasi-hyperbolic discounting Now consider an agent with order-two quasi-hyperbolic preferences. His Euler equation will be given by

$$
u_{x}^{t}-\left(h_{k}^{t+1} u_{x}^{t+1}\right) R \beta \delta \gamma-\delta R\left(1-h_{k}^{t+1}\right)\left[u_{x}^{t+1}+(1-\gamma) \gamma \beta \delta R h_{k}^{t+2} u_{x}^{t+2}\right]=0 .
$$

Turning to our example with $u(x)=\left(x^{1-\sigma}-1\right) /(1-\sigma)$, we again guess that equilibrium consumption is proportional to the available capital: $x^{t}=\lambda_{2} k^{t}$. Substituting this conjecture in (46) gives us an equation for $\lambda_{2}$ :

$$
1-\frac{\lambda_{2} R^{1-\sigma} \beta \delta \gamma}{\left(1-\lambda_{2}\right)^{\sigma}}-\frac{R^{1-\sigma} \delta\left(1-\lambda_{2}\right)}{\left(1-\lambda_{2}\right)^{\sigma}}\left[1-\frac{(1-\gamma) \gamma \beta \delta R^{1-\sigma} \lambda_{2}}{\left(1-\lambda_{2}\right)^{\sigma}}\right]=0
$$

In the limit case when $u(x)=\ln (x)$, solving for $\lambda_{2}$ yields

$$
\lambda_{2}=\frac{1-\delta}{1-\delta[1-\beta \gamma-(1-\gamma) \gamma \beta \delta]}
$$

As already discussed, order-one quasi-hyperbolic discounting is nested as a special case of our ordertwo specification which arises when $\gamma=1$. Not surprisingly, if we set $\gamma=1$, condition (53) reduces to $(52)$.

Order-three quasi-hyperbolic discounting When the agent's preferences are order-three quasi-hyperbolic, his Euler equation takes the form

$$
u_{x}^{t}-R\left\{\gamma+\delta\left[1-(1-\beta) h_{k}^{t+1}\right]\right\} u_{x}^{t+1}+\gamma \delta R^{2}\left(1-h_{k}^{t+1}\right) u_{x}^{t+2}=0 .
$$


If $u(x)=\left(x^{1-\sigma}-1\right) /(1-\sigma)$, we guess a consumption strategy with form $x^{t}=\lambda_{3} k^{t}$. The strategy parameter $\lambda_{3}$ solves the equation

$$
1-\frac{R^{1-\sigma}\left\{\gamma+\delta\left[1-\lambda_{3}(1-\beta)\right]\right\}}{\left(1-\lambda_{3}\right)^{\sigma}}+\frac{\gamma \delta R^{2-2 \sigma}}{\left(1-\lambda_{3}\right)^{2 \sigma-1}}=0
$$

In the limit case when $u(x)=\ln (x)$, we obtain

$$
\lambda_{3}=\frac{(1-\gamma)(1-\delta)}{1-\delta(1-\beta)} .
$$

When $\gamma=0,(54)$ reduces to $(52)$.

True hyperbolic discounting Finally, consider an agents who is a true hyperbolic discounter. As mentioned earlier, a general solution to this problem is not available. However, we could use the method of Calcott and Petkov (2015) to compute the MPE strategy when $u(x)$ is homogeneous of some degree. In particular, suppose that $u(x)=x^{1-\sigma} /(1-\sigma)$. This function is homogeneous of degree $1-\sigma$, implying a consumption strategy $x=\lambda_{h} k$. Substitution in (51) gives us an equation for $\lambda_{h}$ :

$$
\lambda_{h}=\frac{d}{\Phi\left(\left(R\left(1-\lambda_{h}\right)\right)^{1-\sigma}, 1,1 / d\right)},
$$

where $\Phi$ is the Lerch transcendent function as defined by (50).

Numerical comparison of strategies Now we compute numerical examples using the calibrated parameters of order-one, order-two and order-three quasi-hyperbolic preferences presented in Table 1. Our objective is to verify whether the different specifications will make different predictions about agent's consumption strategies. We also compute the MPE strategy for decision makers with true hyperbolic discounting using condition (55). All agents are assumed to have instantaneous 
utility $u(x)=x^{1-\sigma} /(1-\sigma)$. Let $R=1.05$ and $\sigma=0.3$. Table 3 presents the consumption strategy parameters $\lambda_{h}, \lambda_{1}, \lambda_{2}, \lambda_{3}$ for various values of the parameter $d$ of the true hyperbolic discount function (2). The table shows a significant difference between the parameters of the equilibrium strategies with order-one discounting on one hand, and order-two and order-three discounting on the other. These differences are bigger for lower values of $d$. Moreover, $\lambda_{2}$, and especially $\lambda_{3}$, are much closer to $\lambda_{h}$ as compared to $\lambda_{1}$. Thus, our order-two and order-three approximations make predictions that are much more in line with those of the model with true hyperbolic discounting.

\subsection{Application two: smoking and internality taxes}

It has been suggested that internalities due to present-biased preferences are an important reason for government intervention in the market for cigarettes (Gruber and Kozsegi, 2001). Imperfect selfcontrol would cause smokers who cannot commit to future plans to over-consume (as assessed with current preferences over future consumption). This over-consumption takes place because, from the current viewpoint, the smoker's subsequent selves will discount the harm from their addiction too heavily. Taxing cigarettes would reduce smoking by decreasing the marginal utility of cigarettes, but may also provide consumers with a commitment device to help them deal with their self-control problem. In this subsection, we first characterise the smokers' laissez-faire consumption strategies, and then derive corrective taxes that would incentivise socially optimal consumption.

\subsubsection{Setting}

Consider a representative smoker with concave instantaneous utility $u^{t}=v\left(x^{t}, k^{t}\right)-p x^{t}$, where $x^{t}$ is the number of cigarettes smoked in period $t$, and $p$ is the price of a cigarette. The variable $k^{t}$ captures past smoking, and will be referred to as the consumer's "addiction stock". Its law of 
motion of is as follows:

$$
k^{t+1}=\theta k^{t}+x^{t} .
$$

The parameter $\theta$ reflects the persistence of $k$ over time. Smoking is enjoyable, $v_{x}>0$, but it contributes to subsequent addiction. Addiction increases the marginal utility of smoking: $v_{x k}>0$. However, it is harmful to consumers: $v_{k}<0$.

Assume that $v(x, k)$ is homogeneous of degree 1. That is, $v(s x, s k)=s v(x, k)$ for any $s>0$. This property suggests an equilibrium strategy with form $x=\lambda k$. Note that the derivatives $v_{x}$ and $v_{k}$ are homogeneous of degree 0 . Hence, on the equilibrium path marginal utilities will be constant: $v_{x}(\lambda k, k)=v_{x}(\lambda, 1)$ and $v_{k}(\lambda k, k)=v_{k}(\lambda, 1)$.

This application is a special case of the general intra-personal game studied in Section 6 . We can apply the analysis of that section by setting $u(x, k)=v(x, k)-p x$ and $a=\theta, b=1$.

\subsubsection{Laissez-faire consumption strategies}

First suppose that the market for cigarettes is free of government intervention. We now characterise the equilibrium consumption strategies implied by the various time preference specifications.

Smokers with exponential preferences As a benchmark, suppose that the representative smoker's time preferences are exponential, i.e. his discount function is given by (1). As discussed before, these preferences do not give rise to time consistency problems. In the absence of other distortions, consumption will be socially efficient. Setting $a=\theta, b=1$ in Euler equation (31) yields

$$
u_{x}+\delta u_{k}^{t+1}-\delta \theta u_{x}^{t+1}=0 .
$$


We conjecture that the equilibrium consumption strategy is $x=\lambda_{0} k$. This conjecture, together with the homogeneity assumption about $v$, imply the following form for the above condition:

$$
v_{x}\left(\lambda_{0}, 1\right)-p=-\frac{\delta v_{k}\left(\lambda_{0}, 1\right)}{1-\delta \theta}
$$

From this equation we can compute the equilibrium $\lambda_{0}$.

Smokers with order-one quasi-hyperbolic preferences Next consider a smoker with $(\beta, \delta)$ discounting. This specification was assumed by Gruber and Kozsegi (2001) in their work on internality taxes. In that setting, the agent's Euler equation from Section 6 takes the form

$$
u_{x}^{t}+\beta \delta u_{k}^{t+1}-\delta\left[\theta+(1-\beta) h_{k}^{t+1}\right] u_{x}^{t+1}=0 .
$$

As $v$ is homogeneous of degree 1, we conjecture an equilibrium of the smoker's intra-personal game in which agents use strategies $x=\lambda_{1} k$. Then the smoker's generalised Euler equation (58) becomes:

$$
v_{x}\left(\lambda_{1}, 1\right)-p=-\frac{\delta \beta v_{k}\left(\lambda_{1}, 1\right)}{1-\delta\left[\theta+(1-\beta) \lambda_{1}\right]}
$$

We can use the above condition to compute $\lambda_{1}$.

Smokers with order-two quasi-hyperbolic preferences Now imagine that the smoker's time preferences are order-two quasi-hyperbolic. Given $a=\theta, b=1$, addictive consumption will satisfy the following version of Euler equation (41):

$$
u_{x}^{t}+\beta \gamma \delta\left(h_{k}^{t+1} u_{x}^{t+1}+u_{k}^{t+1}\right)-\delta\left(\theta+h_{k}^{t+1}\right)\left[u_{x}^{t+1}+(1-\gamma) \gamma \delta \beta\left(h_{k}^{t+2} u_{x}^{t+2}+u_{k}^{t+2}\right)\right]=0 .
$$


Note that when $\gamma=1$ this equation reduces to (59). Moreover, payoff homogeneity of degree 1 presupposes an equilibrium strategy with form $x=\lambda_{2} k$. Substitution in the above Euler equation gives us the following condition:

$$
v_{x}\left(\lambda_{2}, 1\right)-p=-\frac{\beta \gamma \delta\left[1-\delta\left(\theta+\lambda_{2}\right)(1-\gamma)\right] v_{k}\left(\lambda_{2}, 1\right)}{1-\delta\left[\theta+\lambda_{2}(1-\beta \gamma)\right]-\delta\left(\theta+\lambda_{2}\right)(1-\gamma) \gamma \delta \beta \lambda_{2}}
$$

This condition allows us to compute $\lambda_{2}$.

Smokers with order-three quasi-hyperbolic preferences If the smoker's discount function is third-order quasi-hyperbolic, substituting $a=\theta, b=1$ in Euler equation (46) would give us

$$
u_{x}^{t}+\beta \delta u_{k}^{t+1}-\delta\left[\theta+(1-\beta) h_{k}^{t+1}\right] u_{x}^{t+1}-\gamma\left[\theta u_{x}^{t+1}-u_{k}^{t+1}-\delta\left(\theta+h_{k}^{t+1}\right)\left(\theta u_{x}^{t+2}-u_{k}^{t+2}\right)\right]=0 .
$$

When $\gamma=0$, this equation is equivalent to (59). Moreover, if $v$ is homogeneous of degree 1 , the MPE strategy will have a form $x=\lambda_{3} k$. Hence, the above Euler equation will become

$$
v_{x}\left(\lambda_{3}, 1\right)-p=-\frac{\left\{\beta \delta+\gamma\left[1+\delta\left(\theta+\lambda_{3}\right)\right]\right\} v_{k}\left(\lambda_{3}, 1\right)}{1-\delta\left[\theta+(1-\beta) \lambda_{3}\right]-\gamma \theta\left[1+\delta\left(\theta+\lambda_{3}\right)\right]}
$$

This equation can be solved for $\lambda_{3}$.

Smokers with true hyperbolic preferences Finally, suppose that smokers have true hyperbolic preferences. We can use Calcott and Petkov (2015) to characterise the MPE for the special case when $v$ is homogeneous of degree 1 . As usual, the equilibrium strategy will be proportional to the stock variable: $x=\lambda_{h} k$. Substitution in (51) yields

$$
v_{x}\left(\lambda_{h}, 1\right)-p+\frac{\phi\left(\lambda_{h}\right)\left[v\left(\lambda_{h}, 1\right)-\lambda p\right]}{\left(\lambda_{h}+\theta\right)}=0,
$$


where

$$
\phi\left(\lambda_{h}\right)=\frac{\Phi\left(\left(\lambda_{h}+\theta\right), 1,1 / d\right)}{d}-1 .
$$

Euler's formula implies that

$$
v\left(\lambda_{h}, 1\right)-\lambda p=\left[v_{x}\left(\lambda_{h}, 1\right)-p\right] \lambda+v_{k}\left(\lambda_{h}, 1\right) .
$$

Substituting this decomposition in the above necessary condition gives us

$$
v_{x}\left(\lambda_{h}, 1\right)-p=-\frac{\phi\left(\lambda_{h}\right) v_{k}\left(\lambda_{h}, 1\right)}{\lambda_{h}+\theta+\lambda_{h} \phi\left(\lambda_{h}\right)}=0 .
$$

\subsubsection{Internality taxes}

As already discussed, present-biased preferences will drive smokers to overconsume in the future as assessed from their current viewpoint. This phenomenon is referred to as an "internality". The literature has argued that internalities account for a substantial part of cigarette taxes (Gruber and Kozsegi, 2001). Next we derive the tax policies that induce efficient consumption under the various specifications of the smoker's time preferences.

Additional assumptions Suppose that a benevolent social planner intervenes in the market for cigarettes. He has a single instrument: a per-unit tax $\psi$ levied on each cigarette smoked. All tax revenues are returned to consumers as lump sum transfers. The planner's objective is to maximise lifetime social welfare.

To simplify the analysis, we will assume away all other distortions in the tobacco industry. In particular, imagine that the market is perfectly competitive. That is, the price of cigarettes is equal to the unit production cost: $p=c$. Moreover, we assume that smoking does not give rise to any 
externalities, so the social cost of cigarettes is also $c$. Thus, instantaneous period- $t$ welfare is

$$
\omega^{t}=\omega\left(x^{t}, k^{t}\right)=v\left(x^{t}, k^{t}\right)-c x^{t} .
$$

The planner does not have any time consistency problem: he discounts future welfare exponentially by a factor $\hat{\delta}$. Thus, lifetime welfare from the period- $t$ perspective is given by

$$
\Upsilon^{t}=\sum_{\tau=0}^{\infty}(\hat{\delta})^{t} \omega^{\tau+t}
$$

As before, we will assume that $v$ is homogeneous of degree 1. This assumption implies that the marginal utilities $v_{x}, v_{k}$ are constant on the efficient path. Consequently, it will be possible to implement efficiency with a tax rate that is constant over time.

Efficient consumption rule The efficient consumption plan maximises lifetime welfare (64). It can be described in terms of a rule that assigns a level of consumption to any given value of the state variable $k$. If the above assumptions are satisfied, this consumption rule will have a form $x=\hat{\lambda} k$. The parameter $\hat{\lambda}$ solves (56) where $p=c$ and $\delta=\hat{\delta}$ :

$$
v_{x}(\hat{\lambda}, 1)-c=-\frac{\hat{\delta} v_{k}(\hat{\lambda}, 1)}{1-\hat{\delta} \theta}
$$

Tax for smokers with exponential preferences First suppose that the smoker has exponential preferences. As long as his discount factor is the same as the planner's, he will follow the efficient consumption rule. The objectives of the smoker and the social planner will be identical, so a corrective tax is not necessary: $\psi_{0}=0$. This result is in line with the classic theory of rational addiction, whose supporters claim that market efficiency eliminates the need to tax cigarette consumption. 
Tax for smokers with order-one quasi-hyperbolic preferences Now let the smoker have $(\beta, \delta)$ discounting. If $\beta<1$, this smoker will suffer from present bias. Again, perfect competition implies that $p=c$. We would like to find the tax rate $\psi_{1}$ which generates the efficient consumption rule $\hat{\lambda} k$ as his private MPE strategy, i.e. as a solution to Euler equation (58). The tax reduces the marginal utility of cigarettes: it is now given by $v_{x}(\hat{\lambda}, 1)-c-\psi_{1}$. Replacing the left-hand side of the smoker's private Euler equation (59) with this after-tax marginal utility yields the following condition:

$$
v_{x}(\hat{\lambda}, 1)-c-\psi_{1}=\frac{\delta \beta v_{k}(\hat{\lambda}, 1)}{1-\delta[\theta+(1-\beta) \hat{\lambda}]}
$$

Reconciling this condition with (65) delivers an expression for the efficiency inducing tax rate

$$
\psi_{1}=-\frac{\delta v_{k}(\hat{\lambda}, 1)}{1-\hat{\delta} \theta}+\frac{\delta \beta v_{k}(\hat{\lambda}, 1)}{1-\delta[\theta+(1-\beta) \hat{\lambda}]}
$$

The right-hand side of this expression is the difference between two terms. The first term represents the social valuation of the lifetime harm of smoking an extra cigarette. The second term captures the private valuation of that lifetime harm.

Tax for smokers with order-two quasi-hyperbolic preferences Next consider a smoker with order-two quasi-hyperbolic preferences. Suppose that the government levies a per-unit tax $\psi_{2}$ which implements the efficient consumption rule $\hat{\lambda} k$ as the MPE strategy of the smoker's intrapersonal game. Substituting the after-tax marginal utility of a cigarette in Euler equation (61) suggests that $\psi_{2}$ must satisfy

$$
v_{x}(\hat{\lambda}, 1)-c-\psi_{2}=-\frac{\beta \gamma \delta[1-(\theta+\hat{\lambda})(1-\gamma) \delta] v_{k}(\hat{\lambda}, 1)}{1-\delta[\theta+\hat{\lambda}(1-\beta \gamma)]-\delta(\theta+\hat{\lambda})(1-\gamma) \gamma \delta \beta \hat{\lambda}}
$$


Reconciling this with the efficiency condition (65) yields

$$
\psi_{2}=-\frac{\delta v_{k}(\hat{\lambda}, 1)}{1-\hat{\delta} \theta}+\frac{\beta \gamma \delta[1-\delta(\theta+\hat{\lambda})(1-\gamma)] v_{k}(\hat{\lambda}, 1)}{1-\delta[\theta+\hat{\lambda}(1-\beta \gamma)]-\delta(\theta+\hat{\lambda})(1-\gamma) \gamma \delta \beta \hat{\lambda}}
$$

As before, the efficiency inducing tax rate is equal to the difference between the social and the private valuations of the marginal harm of smoking an extra cigarette. However, the private valuation is now given by

$$
-\frac{\beta \gamma \delta[1-\delta(\theta+\hat{\lambda})(1-\gamma)] v_{k}(\hat{\lambda}, 1)}{1-\delta[\theta+\hat{\lambda}(1-\beta \gamma)]-\delta(\theta+\hat{\lambda})(1-\gamma) \gamma \delta \beta \hat{\lambda}} .
$$

This expression accounts for the modifications introduced to the smoker's discount function. In the special case when $\gamma=1$ we have $\psi_{2}=\psi_{1}$.

Tax for smokers with order-three quasi-hyperbolic preferences Imagine that a smoker is an order-three quasi-hyperbolic discounter. Let the government's per-unit tax now be $\psi_{3}$. To implement $\hat{\lambda} k$ as the MPE strategy of the smoker's intra-personal game, $\psi_{3}$ must be such that $\hat{\lambda}$ solves Euler equation (63) with marginal utility of cigarettes set to $v_{x}(\hat{\lambda}, 1)-c-\psi_{3}$. That is, the tax rate must satisfy

$$
v_{x}(\hat{\lambda}, 1)-c-\psi_{3}=-\frac{\{\delta \beta+\gamma[1-\delta(\theta+\hat{\lambda})]\} v_{k}(\hat{\lambda}, 1)}{1-\delta[\theta+(1-\beta) \hat{\lambda}]-\gamma \theta[1-\delta(\theta+\hat{\lambda})]}
$$

Reconciling this condition with (65) gives us

$$
\psi_{3}=-\frac{\hat{\delta} v_{k}(\hat{\lambda}, 1)}{1-\hat{\delta} \theta}+\frac{\{\delta \beta+\gamma[1-\delta(\theta+\hat{\lambda})]\} v_{k}(\hat{\lambda}, 1)}{1-\delta[\theta+(1-\beta) \hat{\lambda}]-\gamma \theta[1-\delta(\theta+\hat{\lambda})]}
$$


The difference with the tax rates derived previously is in the private valuation of the lifetime harm of smoking an extra cigarette. Now this valuation has a form

$$
-\frac{\{\delta \beta+\gamma[1-\delta(\theta+\hat{\lambda})]\} v_{k}(\hat{\lambda}, 1)}{1-\delta[\theta+(1-\beta) \hat{\lambda}]-\gamma \theta[1-\delta(\theta+\hat{\lambda})]} .
$$

Tax for smokers with order-three quasi-hyperbolic preferences Finally we study implementation of efficiency when smokers have true hyperbolic preferences. If the government imposes a tax rate of $\psi_{h}$, the private optimum will be consistent with efficiency if

$$
v_{x}(\hat{\lambda}, 1)-c-\psi_{h}=-\frac{\phi(\hat{\lambda}) v_{k}(\hat{\lambda}, 1)}{\hat{\lambda}+\theta+\hat{\lambda} \phi(\hat{\lambda})}
$$

where $\phi(\hat{\lambda})$ is as defined earlier. Reconciliation with (65) suggests that the expression for the efficiency inducing tax rate in this case would be given by

$$
\psi_{h}=-\frac{\hat{\delta} v_{k}(\hat{\lambda}, 1)}{1-\hat{\delta} \theta}+\frac{\phi(\hat{\lambda}) v_{k}(\hat{\lambda}, 1)}{\hat{\lambda}+\theta+\hat{\lambda} \phi(\hat{\lambda})} .
$$

With true hyperbolic discounting, the private valuation of the lifetime harm from consuming an extra cigarette is

$$
\frac{\phi(\hat{\lambda}) v_{k}(\hat{\lambda}, 1)}{\hat{\lambda}+\theta+\hat{\lambda} \phi(\hat{\lambda})} .
$$

Numerical examples To quantify the implications of the generalised quasi-hyperbolic preferences for addictive consumption and internality taxes, we now study a numerical example. Suppose that the smoker's instantaneous utility is

$$
v(x, k)=x^{\alpha} k^{1-\alpha}-r k .
$$


Note that this utility satisfies the requirements of our model. The implied marginal utilities are

$$
v_{x}(\lambda, 1)=\alpha \lambda^{\alpha-1}, \quad v_{k}(\lambda, 1)=(1-\alpha) \lambda^{\alpha}-r .
$$

We can substitute these expressions in the Euler equations of Section 7.2.2 and Section 7.2.3. To compute the numerical examples, we need to assign specific values to the parameters of the model. Let $\theta=0.5$, and let the social planner's discount factor be $\hat{\delta}=0.9$. Moreover, let $\alpha=0.5, c=$ $1, r=1$. Table 4 reports the efficiency inducing tax rate for true hyperbolic consumers, as well as the tax rates for the various quasi-hyperbolic approximations as calibrated in Section 5. The table demonstrates that our order-two and order-three approximations yield tax rates that are close to the optimal tax when discounting is true hyperbolic. Our policy predictions are much more accurate relative to standard $(\beta, \delta)$ discounting.

\section{Applications in which an exact solution is not available}

In the two applications studied above, it was possible to obtain an exact solution for the equilibrium strategies of agents with true hyperbolic preferences. Thus, there was no need to actually approximate true hyperbolic discounting with alternative specifications. The equilibria for settings with quasi-hyperbolic discounting were computed to gauge the precision of the policy predictions generated with these approximations.

However, in many instances exact solutions to problems of agents with true hyperbolic discounting are simply not available. The assumptions used by Calcott and Petkov (2015) to derive such solutions are quite restrictive. Specifically, instantaneous utilities must be homogeneous of degree 1. Any other specification would typically yield infinite lifetime payoffs. Moreover, instead of using dynamic programming techniques, the analysis of Calcott and Petkov (2015) relied on the one-shot 
deviation principle. But sometimes it may be necessary to formulate a problem recursively in order to solve it.

In this section, we will consider two models which are not solvable when preferences are true hyperbolic. Since an exact solution cannot be obtained, we have to resort to approximations. We argue that in such cases our approach is the best alternative. It usually yields finite lifetime payoffs, and is amenable to recursive formulation. The analytical and computational tractability of our method is reasonable and comparable to that of Laibson's.

\subsection{A model with Markovian uncertainty}

First we study a problem with a stochastic component. In particular, we consider a version of our second application in which the smoker's instantaneous utility is random: it follows a Markovian process. Because of this Markovian structure, we need to formulate the problem recursively in order to solve it. While such a formulation is not possible for agents with true hyperbolic discounting, it is quite feasible with our order-two and order-three quasi-hyperbolic approximations.

\subsubsection{Setting}

Consider a smoker with the following utility function:

$$
v=x^{\alpha} k^{1-\alpha}-r k .
$$

The parameter $r$ is now a random variable that follows a Markovian chain. Suppose that $r$ can take two values: $r_{s}$ and $r_{b}$. If $r^{t}=r_{s}$, then with probability $p_{s}$ we will have $r^{t+1}=r_{s}$, and with probability $p_{b}$ (where $p_{b}=1-p_{s}$ ) we will have $r^{t+1}=r_{b}$. Similarly, if $r^{t}=r_{b}$, then $r^{t+1}=r_{s}$ with probability $q_{s}$, and $r^{t+1}=r_{b}$ with probability $q_{b}=1-q_{s}$. Note that the state of the world is now two-dimensional: it is described by $k$ and $r$. All other assumptions are the same as in the setting 
of Section 7.2. As before, we focus on a perfectly competitive industry, so in each period $p=c$.

\subsubsection{Exponential discounting}

Suppose that a social planner can directly choose the smoker's consumption. In period $t$, instantaneous welfare is given by

$$
w^{t}=\left(x^{t}\right)^{\alpha}\left(k^{t}\right)^{1-\alpha}-r k^{t}-c x^{t}
$$

where $r$ is as defined above. Again, for simplicity we will assume that the social planner is an exponential discounter. Let his discount factor be $\hat{\delta}$. As $r$ is now part of the state, the strategies and the value functions will depend on the realisation of $r$. Let $\Upsilon_{s}\left(k^{t}\right)$ be the planner's value function when $r^{t}=r_{s}$, and let $\Upsilon_{b}\left(k^{t}\right)$ be his value function when $r^{t}=r_{b}$. The planner's Bellman equations are

$$
\begin{aligned}
& \Upsilon_{s}\left(k^{t}\right)=\max _{x^{t}}\left\{\left(x^{t}\right)^{\alpha}\left(k^{t}\right)^{1-\alpha}-r_{s} k^{t}-c x^{t}+\hat{\delta}\left[p_{s} \Upsilon_{s}\left(x^{t}+\theta k^{t}\right)+p_{b} \Upsilon_{b}\left(x^{t}+\theta k^{t}\right)\right]\right\}, \\
& \Upsilon_{b}\left(k^{t}\right)=\max _{x^{t}}\left\{\left(x^{t}\right)^{\alpha}\left(k^{t}\right)^{1-\alpha}-r_{b} k^{t}-c x^{t}+\hat{\delta}\left[q_{s} \Upsilon_{s}\left(x^{t}+\theta k^{t}\right)+q_{b} \Upsilon_{b}\left(x^{t}+\theta k^{t}\right)\right]\right\} .
\end{aligned}
$$

Note that instantaneous welfare is homogeneous of degree 1 . Thus, we conjecture optimal consumption policies with forms $x^{t}=\lambda_{s} k^{t}$ when $r^{t}=r_{s}$ and $x^{t}=\lambda_{b} k^{t}$ when $r^{t}=r_{b}$. This, in turn, suggests linear value functions: $\Upsilon_{s}\left(k^{t}\right)=\Upsilon_{s} k^{t}$ and $\Upsilon_{b}\left(k^{t}\right)=\Upsilon_{b} k^{t}$. Evaluating the Bellman equations (67), (68) on the optimal path gives us:

$$
\begin{aligned}
& \Upsilon_{s}=\left(\lambda_{s}\right)^{\alpha}-r_{s}-c \lambda_{s}+\left(\lambda_{s}+\theta\right) \hat{\delta}\left[p_{s} \Upsilon_{s}+p_{b} \Upsilon_{b}\right] \\
& \Upsilon_{b}=\left(\lambda_{b}\right)^{\alpha}-r_{b}-c \lambda_{b}+\left(\lambda_{b}+\theta\right) \hat{\delta}\left[q_{s} \Upsilon_{s}+q_{b} \Upsilon_{b}\right]
\end{aligned}
$$


Furthermore, differentiating (67), (68) with respect to $x^{t}$ yields the following first-order conditions:

$$
\begin{aligned}
& \alpha\left(x^{t}\right)^{\alpha-1}\left(k^{t}\right)^{1-\alpha}-c+\hat{\delta}\left[p_{s} \Upsilon_{s}+p_{b} \Upsilon_{b}\right]=0, \\
& \alpha\left(x^{t}\right)^{\alpha-1}\left(k^{t}\right)^{1-\alpha}-c+\hat{\delta}\left[q_{s} \Upsilon_{s}+q_{b} \Upsilon_{b}\right]=0 .
\end{aligned}
$$

If we evaluate these first-order conditions at $x=\lambda_{s} k$ and $x=\lambda_{b} k$, respectively, we obtain

$$
\begin{aligned}
& \alpha\left(\lambda_{s}\right)^{\alpha-1}-c+\hat{\delta}\left[p_{s} \Upsilon_{s}+p_{b} \Upsilon_{b}\right]=0 . \\
& \alpha\left(\lambda_{b}\right)^{\alpha-1}-c+\hat{\delta}\left[q_{s} \Upsilon_{s}+q_{b} \Upsilon_{b}\right]=0 .
\end{aligned}
$$

Equations (71), (72), together with (69), (70), enable us to compute the parameters $\lambda_{s}, \lambda_{b}$ of the socially optimal consumption policies, as well as the coefficients $\Upsilon_{s}, \Upsilon_{b}$ of the planner's value functions.

To illustrate the properties of the model, we compute a numerical example. Suppose that $\alpha=0.5, r_{1}=1, r_{2}=2, \theta=0.5, c=0, \hat{\delta}=0.95$. Table 5 shows the optimal consumption policies for various values of the transition probabilities $p_{s}, q_{s}$.

\subsubsection{Order-two discounting}

Imagine that smoker has an order-two quasi-hyperbolic discount function. We can use equations (13), (14), (15) from Section 3 to formulate his problem recursively. As already noted, $r$ is now an element of the state. Thus, we need to specify two current value functions. Let $U_{s}\left(k^{t}\right)$ be the current lifetime payoff when $r^{t}=r_{s}$, and let $U_{b}\left(k^{t}\right)$ be the current lifetime payoff when $r^{t}=r_{b}$. Similarly, let $V_{s}\left(k^{t}\right)$ be the consumer's continuation payoff when $r^{t}=r_{s}$, and let $V_{b}\left(k^{t}\right)$ be his continuation payoff when $r^{t}=r_{b}$. Finally, let $W_{s}\left(k^{t}\right)$ be the third value function when $r^{t}=r_{s}$, and let $W_{b}\left(k^{t}\right)$ be the third value function when $r^{t}=r_{b}$. We will also assume that smoking incurs a per 
unit tax of $\psi_{s}$ when $r^{t}=r_{s}$ and a per-unit tax of $\psi_{b}$ when $r^{t}=r_{b}$. The smoker's MPE strategies are $h_{s}\left(k^{t}\right)$ and $h_{b}\left(k^{t}\right)$, respectively.

In this setting, the consumer's Bellman equations can be written as follows:

$$
\begin{aligned}
U_{s}\left(k^{t}\right)= & \max _{x^{t}}\left\{\left(x^{t}\right)^{\alpha}\left(k^{t}\right)^{1-\alpha}-r_{s} k^{t}-\left(c+\psi_{s}\right) x^{t}\right. \\
& \left.+\beta \gamma \delta\left[p_{s} V_{s}\left(x^{t}+\theta k^{t}\right)+p_{b} V_{b}\left(x^{t}+\theta k^{t}\right)\right]\right\}, \\
U_{b}\left(k^{t}\right)= & \max _{x^{t}}\left\{\left(x^{t}\right)^{\alpha}\left(k^{t}\right)^{1-\alpha}-r_{b} k^{t}-\left(c+\psi_{b}\right) x^{t}\right. \\
& \left.+\beta \gamma \delta\left[q_{s} V_{s}\left(x^{t}+\theta k^{t}\right)+q_{b} V_{b}\left(x^{t}+\theta k^{t}\right)\right]\right\} .
\end{aligned}
$$

The continuation payoffs $V_{s}\left(k^{t}\right)$ and $V_{b}\left(k^{t}\right)$ solve the functional equations

$$
\begin{aligned}
V_{s}\left(k^{t}\right)= & \left(h_{s}\left(k^{t}\right)\right)^{\alpha}\left(k^{t}\right)^{1-\alpha}-r_{s} k^{t}-\left(c+\psi_{s}\right) h_{s}\left(k^{t}\right) \\
& +\gamma \delta\left[p_{s} W\left(h_{s}\left(k^{t}\right)+\theta k^{t}\right)+p_{b} W_{b}\left(h_{s}\left(k^{t}\right)+\theta k^{t}\right)\right], \\
V_{b}\left(k^{t}\right)= & \left(h_{b}\left(k^{t}\right)\right)^{\alpha}\left(k^{t}\right)^{1-\alpha}-r_{b} k^{t}-\left(c+\psi_{b}\right) h_{b}\left(k^{t}\right) \\
& +\gamma \delta\left[q_{s} W_{s}\left(h_{b}\left(k^{t}\right)+\theta k^{t}\right)+q_{b} W_{b}\left(h_{b}\left(k^{t}\right)+\theta k^{t}\right)\right],
\end{aligned}
$$

where $W_{s}\left(k^{t}\right)$ and $W_{b}\left(k^{t}\right)$ satisfy

$$
\begin{aligned}
W_{s}\left(k^{t}\right)= & \left(h_{s}\left(k^{t}\right)\right)^{\alpha}\left(k^{t}\right)^{1-\alpha}-r_{s} k^{t}-\left(c+\psi_{s}\right) h_{s}\left(k^{t}\right) \\
& +\delta\left[p_{s} W_{s}\left(h_{s}\left(k^{t}\right)+\theta k^{t}\right)+p_{b} W_{b}\left(h_{s}\left(k^{t}\right)+\theta k^{t}\right)\right], \\
W_{b}\left(k^{t}\right)= & \left(h_{b}\left(k^{t}\right)\right)^{\alpha}\left(k^{t}\right)^{1-\alpha}-r_{b} k^{t}-\left(c+\psi_{b}\right) h_{b}\left(k^{t}\right) \\
& +\delta\left[q_{s} W_{s}\left(h_{b}\left(k^{t}\right)+\theta k^{t}\right)+q_{b} W_{b}\left(h_{b}\left(k^{t}\right)+\theta k^{t}\right)\right] .
\end{aligned}
$$

Given that payoffs are homogeneous of degree 1, the equilibrium will involve strategies $x^{t}=$ 
$\lambda_{s} k^{t}$ when $r^{t}=r_{s}$ and $x^{t}=\lambda_{b} k^{t}$ when $r^{t}=r_{b}$. These strategies imply linear value functions: $U_{s}\left(k^{t}\right)=U_{s} k^{t}, U_{b}\left(k^{t}\right)=U_{b} k^{t}, V_{s}\left(k^{t}\right)=V_{s} k^{t}, V_{b}\left(k^{t}\right)=V_{b} k^{t}, W_{s}\left(k^{t}\right)=W_{s} k^{t}, W_{b}\left(k^{t}\right)=W_{b} k^{t}$. Thus, if we evaluate the Bellman equations on the equilibrium path, we will obtain

$$
\begin{aligned}
& U_{s}=\left(\lambda_{s}\right)^{\alpha}-r_{s}-\left(c+\psi_{s}\right) \lambda_{s}+\beta \gamma \delta\left(\lambda_{s}+\theta\right)\left[p_{s} V_{s}+p_{b} V_{b}\right], \\
& U_{b}=\left(\lambda_{b}\right)^{\alpha}-r_{b}-\left(c+\psi_{b}\right) \lambda_{b}+\beta \gamma \delta\left(\lambda_{b}+\theta\right)\left[q_{s} V_{s}+q_{b} V_{b}\right] .
\end{aligned}
$$

Moreover, conditions (75), (76) and (77), (78) simplify to

$$
\begin{aligned}
V_{s} & =\left(\lambda_{s}\right)^{\alpha}-r_{s}-\left(c+\psi_{s}\right) \lambda_{s}+\gamma \delta\left(\lambda_{s}+\theta\right)\left[p_{s} W_{s}+p_{b} W_{b}\right], \\
V_{s} & =\left(\lambda_{b}\right)^{\alpha}-r_{b}-\left(c+\psi_{b}\right) \lambda_{b}+\gamma \delta\left(\lambda_{b}+\theta\right)\left[q_{s} W_{s}+q_{b} W_{b}\right]
\end{aligned}
$$

and

$$
\begin{aligned}
& W_{s}=\left(\lambda_{s}\right)^{\alpha}-r_{s}-\left(c+\psi_{s}\right) \lambda_{s}+\delta\left(\lambda_{s}+\theta\right)\left[p_{s} W_{s}+p_{b} W_{b}\right], \\
& W_{s}=\left(\lambda_{b}\right)^{\alpha}-r_{b}-\left(c+\psi_{b}\right) \lambda_{b}+\delta\left(\lambda_{b}+\theta\right)\left[q_{s} W_{s}+q_{b} W_{b}\right],
\end{aligned}
$$

respectively. Finally, differentiating the two Bellman equations yields the following first-order conditions:

$$
\begin{aligned}
& \alpha\left(x^{t}\right)^{\alpha-1}\left(k^{t}\right)^{1-\alpha}-\left(c+\psi_{s}\right)+\beta \gamma \delta\left[p_{s} V_{s}+p_{b} V_{b}\right]=0, \\
& \alpha\left(x^{t}\right)^{\alpha-1}\left(k^{t}\right)^{1-\alpha}-\left(c+\psi_{b}\right)+\beta \gamma \delta\left[q_{s} V_{s}+q_{b} V_{b}\right]=0 .
\end{aligned}
$$


Evaluating these first-order conditions at at $x=\lambda_{s} k$ and $x=\lambda_{b} k$, respectively, we get

$$
\begin{aligned}
\alpha\left(\lambda_{s}\right)^{\alpha-1}-\left(c+\psi_{s}\right)+\beta \gamma \delta\left[p_{s} V_{s}+p_{b} V_{b}\right] & =0, \\
\alpha\left(\lambda_{b}\right)^{\alpha-1}-\left(c+\psi_{b}\right)+\beta \gamma \delta\left[q_{s} V_{s}+q_{b} V_{b}\right] & =0 .
\end{aligned}
$$

For given tax rates $\psi_{s}, \psi_{b}$, conditions (79), (80), (81), (82), (83), (84), (85), (86) allow us to compute the strategy parameters $\lambda_{s}, \lambda_{b}$, as well as the coefficients $U_{s}, U_{b}, V_{s}, V_{b}, W_{s}, W_{b}$ of the value functions.

Now imagine that the social planner sets the tax rates to implement his preferred consumption policies $\lambda_{s}, \lambda_{b}$ characterised in Section 8.1.2 as MPE strategies in the smoker's intra-personal game. Then equations (79), (80), (81), (82), (83), (84), (85) (86) can be used to compute the efficiency inducing taxes $\psi_{s}, \psi_{b}$, as well as the coefficients $U_{s}, U_{b}, V_{s}, V_{b}, W_{s}, W_{b}$ of the value functions. For illustration we compute a numerical example with the same parameter values as in Section 8.1.2. We use the values of $\delta, \beta, \gamma$ from our calibration exercise with $d=0.7: \delta=.89261, \beta=.98796, \gamma=$ .66703. Table 6 shows the laissez-faire consumption strategies $\lambda_{s}, \lambda_{b}$, as well as the optimal tax rates $\psi_{s}, \psi_{b}$.

\subsubsection{Order-three discounting}

Now consider a smoker with order-three quasi-hyperbolic preferences. Let $U_{s}\left(k^{t}\right)$ be the current value function when $r^{t}=r_{s}$, and let $U_{b}\left(k^{t}\right)$ be the current value function when $r^{t}=r_{b}$. Similarly, let $W_{s}\left(k^{t}\right)$ and $W_{b}\left(k^{t}\right)$ be the continuation value functions when $r^{t}=r_{s}$ and $r^{t}=r_{b}$, respectively. As before, smoking incurs a per unit tax of $\psi_{s}$ when $r^{t}=r_{s}$ and a per-unit tax of $\psi_{b}$ when $r^{t}=r_{b}$. The smoker's MPE strategies are $h_{s}\left(k^{t}\right)$ and $h_{b}\left(k^{t}\right)$. 
The recursive formulation (27), (28) from Section 4 implies the following Bellman equations:

$$
\begin{gathered}
U_{s}\left(k^{t}\right)=\max _{x^{t}}\left\{\left(x^{t}\right)^{\alpha}\left(k^{t}\right)^{1-\alpha}-r_{s} k^{t}-\left(c+\psi_{s}\right) x^{t}+\gamma\left[p_{s} U_{s}\left(x^{t}+\theta k^{t}\right)+p_{b} U_{b}\left(x^{t}+\theta k^{t}\right)\right]\right. \\
\left.+\delta \beta\left[p_{s} W_{s}\left(x^{t}+\theta k^{t}\right)+p_{b} W_{b}\left(x^{t}+\theta k^{t}\right)\right]\right\}, \\
U_{b}\left(k^{t}\right)=\max _{x^{t}}\left\{\left(x^{t}\right)^{\alpha}\left(k^{t}\right)^{1-\alpha}-r_{b} k^{t}-\left(c+\psi_{b}\right) x^{t}+\gamma\left[q_{s} U_{s}\left(x^{t}+\theta k^{t}\right)+q_{b} U_{b}\left(x^{t}+\theta k^{t}\right)\right]\right. \\
\left.+\delta \beta\left[q_{s} W_{s}\left(x^{t}+\theta k^{t}\right)+q_{b} W_{b}\left(x^{t}+\theta k^{t}\right)\right]\right\} .
\end{gathered}
$$

The continuation value functions must satisfy

$$
\begin{aligned}
W_{s}\left(k^{t}\right)= & \left(h_{s}\left(k^{t}\right)\right)^{\alpha}\left(k^{t}\right)^{1-\alpha}-r_{s} k^{t}-\left(c+\psi_{s}\right) h_{s}\left(k^{t}\right) \\
& +\delta\left[p_{s} W_{s}\left(h_{s}\left(k^{t}\right)+\theta k^{t}\right)+p_{b} W_{b}\left(h_{s}\left(k^{t}\right)+\theta k^{t}\right)\right], \\
W_{b}\left(k^{t}\right)= & \left(h_{b}\left(k^{t}\right)\right)^{\alpha}\left(k^{t}\right)^{1-\alpha}-r_{b} k^{t}-\left(c+\psi_{b}\right) h_{b}\left(k^{t}\right) \\
& +\delta\left[q_{s} W_{s}\left(h_{b}\left(k^{t}\right)+\theta k^{t}\right)+q_{b} W_{b}\left(h_{b}\left(k^{t}\right)+\theta k^{t}\right)\right] .
\end{aligned}
$$

Again, when $r^{t}=r_{s}$ the smoker's MPE strategy is $x^{t}=\lambda_{s} k^{t}$, and when $r^{t}=r_{b}$ his MPE strategy is $x^{t}=\lambda_{b} k^{t}$. Consequently, the value functions will be linear: $U_{s}\left(k^{t}\right)=U_{s} k^{t}, U_{b}\left(k^{t}\right)=U_{b} k^{t}$ and $W_{s}\left(k^{t}\right)=W_{s} k^{t}, W_{b}\left(k^{t}\right)=W_{b} k^{t}$. Evaluating the Bellman equations (87) and (88) on the equilibrium path gives us:

$$
\begin{aligned}
U_{s} & =\left(\lambda_{s}\right)^{\alpha}-\left(c+\psi_{s}\right) \lambda_{s}-r_{s}+\gamma\left(\lambda_{s}+\theta\right)\left[p_{s} U_{s}+p_{b} U_{b}\right]+\delta \beta\left(\lambda_{s}+\theta\right)\left[p_{s} W_{s}+p_{b} W_{b}\right], \\
U_{b} & =\left(\lambda_{b}\right)^{\alpha}-\left(c+\psi_{b}\right) \lambda_{b}-r_{b}+\gamma\left(\lambda_{b}+\theta\right)\left[q_{s} U_{s}+q_{b} U_{b}\right]+\delta \beta\left(\lambda_{b}+\theta\right)\left[q_{s} W_{s}+q_{b} W_{b}\right] .
\end{aligned}
$$


Moreover, conditions (89) and (90) simplify to

$$
\begin{aligned}
& W_{s}=\left(\lambda_{s}\right)^{\alpha}-\left(c+\psi_{s}\right) \lambda_{s}-r_{s}+\delta\left(\lambda_{s}+\theta\right)\left(p_{s} W_{s}+p_{b} W_{b}\right), \\
& W_{b}=\left(\lambda_{b}\right)^{\alpha}-\left(c+\psi_{b}\right) \lambda_{b}-r_{b}+\delta\left(\lambda_{b}+\theta\right)\left(q_{s} W_{s}+q_{b} W_{b}\right) .
\end{aligned}
$$

Finally, differentiating the Bellman equations (87) and (88) yields the following first-order conditions:

$$
\begin{aligned}
& \alpha\left(x^{t}\right)^{\alpha-1}\left(k^{t}\right)^{1-\alpha}-\left(c+\psi_{s}\right)+\gamma\left[p_{s} U_{s}+p_{b} U_{b}\right]+\delta \beta\left[p_{s} W_{s}+p_{b} W_{b}\right]=0, \\
& \alpha\left(x^{t}\right)^{\alpha-1}\left(k^{t}\right)^{1-\alpha}-\left(c+\psi_{b}\right)+\gamma\left[q_{s} U_{s}+q_{b} U_{b}\right]+\delta \beta\left[q_{s} W_{s}+q_{b} W_{b}\right]=0,
\end{aligned}
$$

Evaluating these first-order conditions at $x^{t}=\lambda_{s} k^{t}$ and $x^{t}=\lambda_{b} k^{t}$, respectively, gives us

$$
\begin{aligned}
& \alpha\left(\lambda_{s}\right)^{\alpha-1}-\left(c+\psi_{s}\right)+\gamma\left[p_{s} U_{s}+p_{b} U_{b}\right]+\delta \beta\left[p_{s} W_{s}+p_{b} W_{b}\right]=0 \\
& \alpha\left(\lambda_{b}\right)^{\alpha-1}-\left(c+\psi_{b}\right)+\gamma\left[q_{s} U_{s}+q_{b} U_{b}\right]+\delta \beta\left[q_{s} W_{s}+q_{b} W_{b}\right]=0
\end{aligned}
$$

Equations (91), (92), (93), (94), (95), (96) can be solved numerically to deliver the MPE strategy parameters $\lambda_{s}, \lambda_{b}$, and the coefficients $U_{s}, U_{b}, W_{s}, W_{b}$ of the value functions.

Again, we could compute the tax rates that implement the optimal consumption policies $\lambda_{s}, \lambda_{b}$ in the example of Section 8.1.2. To do that, we solve (91), (92), (93), (94), (95), (96) for $\psi_{s}, \psi_{b}$, as well as for the coefficients $U_{s}, U_{b}, W_{s}, W_{b}$. Assume the same parameters as in Section 8.1.2. The values of $\delta, \beta, \gamma$ are those that deliver the best fit for true hyperbolic discounting with $d=0.7$ : $\delta=.92671, \beta=.13683, \gamma=.48324$. Table 7 presents the laissez-faire strategies, $\lambda_{s}, \lambda_{b}$, and the optimal tax rates, $\psi_{s}, \psi_{b}$. 


\subsection{Linear-quadratic utility}

We now consider an alternative specification of the decision maker's instantaneous utility. In particular, suppose that a smoker has the following utility function:

$$
u(x, k)=a_{x} x-\frac{a_{x x}}{2} x^{2}+a_{x k} x k-a_{k} k-\frac{a_{k k}}{2} k^{2},
$$

where $a_{x}>0, a_{x x}>0, a_{x k}>0, a_{k}>0, a_{k k}>0$. The corresponding marginal utilities are

$$
\begin{aligned}
& u_{x}\left(x^{t}, k^{t}\right)=a_{x}-a_{x x} x^{t}+a_{x k} k^{t} \\
& u_{k}\left(x^{t}, k^{t}\right)=a_{x k} x^{t}-a_{k}-a_{k k} k^{t} .
\end{aligned}
$$

As before, the addiction stock evolves according to

$$
k^{t+1}=x^{t}+\theta k^{t} .
$$

This payoff specification satisfies the standard assumptions imposed in models of rational addiction.

That is, i) smoking brings instantaneous satisfaction but reduces future utility, and ii) smoking today increases the marginal utility of smoking tomorrow. In fact, this particular functional form was assumed in Becker and Murphy (1988).

Linear-quadratic payoffs usually imply MPE strategies with form

$$
h\left(k^{t}\right)=\lambda k^{t}+\mu .
$$

Thus, per-period payoffs will usually have a non-zero value in the steady state. This suggests that the optimal consumption plan of a true hyperbolic discounter with such utility will not be interior. 
The reason is that his lifetime payoff will be infinite. However, if we want to compute an interior consumption plan in a setting with similar qualitative properties, we could use our order-two and order-three approximations. They would deliver finite lifetime payoffs so long as $\delta<1$.

\subsubsection{Order-two discounting}

Consider a setting in which a consumer has an order-two quasi-hyperbolic discount function. As established earlier, his MPE strategy will satisfy (60), where $u_{x}$ and $u_{k}$ are now given by (98) and (99), respectively. Furthermore, we conjecture that the MPE strategy is $h\left(k^{t}\right)=\lambda_{2} k^{t}+\mu_{2}$. Therefore, $h_{k}=\lambda_{2}$. Consequently, (41) will take the following form:

$$
\begin{gathered}
0=a_{x}-a_{x x} x^{t}+a_{x k} k^{t}+\beta \gamma \delta\left[\lambda_{2}\left(a_{x}-a_{x x} x^{t+1}+a_{x k} k^{t+1}\right)+a_{x k} x^{t+1}-a_{k}-a_{k k} k^{t+1}\right]- \\
\delta\left(\theta+\lambda_{2}\right)\left\{a_{x}-a_{x x} x^{t+1}+a_{x k} k^{t+1}+\gamma \delta \beta(1-\gamma)\left[\lambda_{2}\left(a_{x}-a_{x x} x^{t+2}+a_{x k} k^{t+2}\right)+a_{x k} x^{t+2}-a_{k}-a_{k k} k^{t+2}\right] .\right.
\end{gathered}
$$

Moreover, in equilibrium we must have

$$
\begin{aligned}
k^{t+1} & =\theta k^{t}+x^{t}, \\
x^{t+1} & =\lambda_{2} k^{t+1}+\mu_{2}, \\
k^{t+2} & =\left(\lambda_{2}+\theta\right) k^{t+1}+\mu_{2}, \\
x^{t+2} & =\lambda_{2} k^{t+2}+\mu_{2} .
\end{aligned}
$$

We can substitute (102), (103), (104), (105) in (101) and apply the method of undetermined coefficients to obtain equations for the MPE strategy parameters $\lambda_{2}, \mu_{2}$.

For example, suppose that $a_{x}=1, a_{x x}=2, a_{x k}=1, a_{k}=1, a_{k k}=2$. Let $\theta=0.5$. Moreover, we assign $\beta, \delta, \gamma$ their calibrated values obtained in Section 5 for $d=0.7$. That is, $\delta=.89261, \beta=$ $.98796, \gamma=.66703$. Given these parameters, we compute the following MPE strategy: $\lambda_{2}=.087534$, 
$\mu_{2}=.018435$. The steady state addiction stock $\bar{k}_{2}$ solves $\bar{k}_{2}=\left(\lambda_{2}+\theta\right) \bar{k}_{2}+\mu_{2}$. Thus, $\bar{k}_{2}=.044694$.

Consequently, steady state addictive consumption would be $\bar{x}_{2}=\lambda_{2} \bar{k}_{2}+\mu_{2}=.022347$.

\subsubsection{Order-three discounting}

Now suppose that the consumer is a third-order quasi-hyperbolic discounter. Thus, his equilibrium strategy will satisfy (62). The marginal utilities $u_{x}$ and $u_{k}$ are again given by (98) and (99). As in the previous subsection, we conjecture a linear MPE strategy: $h\left(k^{t}\right)=\lambda_{3} k^{t}+\mu_{3}$. Hence, $h_{k}=\lambda_{3}$. Substituting the derivatives of the utility and the strategy conjecture in the decision maker's generalised Euler equation yields

$$
\begin{gathered}
0=\left(a_{x}-a_{x x} x^{t}+a_{x k} k^{t}\right)+\beta \delta\left(a_{x k} x^{t+1}-a_{k}-a_{k k} k^{t+1}\right)-\delta\left[\theta+(1-\beta) \lambda_{3}\right]\left(a_{x}-a_{x x} x^{t+1}+a_{x k} k^{t+1}\right) \\
-\gamma\left\{\theta\left(a_{x}-a_{x x} x^{t+1}+a_{x k} k^{t+1}\right)-\left(a_{x k} x^{t+1}-a_{k}-a_{k k} k^{t+1}\right)\right. \\
+\gamma \delta\left(\theta+\lambda_{3}\right)\left\{\theta\left(a_{x}-a_{x x} x^{t+2}+a_{x k} k^{t+2}\right)-\left(a_{x k} x^{t+2}-a_{k}-a_{k k} k^{t+2}\right)\right\},
\end{gathered}
$$

where

$$
\begin{aligned}
k^{t+1} & =\theta k^{t}+x^{t}, \\
x^{t+1} & =\lambda_{3} k^{t+1}+\mu_{3}, \\
k^{t+2} & =\left(\lambda_{3}+\theta\right) k^{t+1}+\mu_{3}, \\
x^{t+2} & =\lambda_{3} k^{t+2}+\mu_{3} .
\end{aligned}
$$

Applying the method of the undetermined coefficients to the above condition gives us equations for the strategy parameters $\lambda_{3}, \mu_{3}$.

Again, consider the numerical example with utility parameters $a_{x}=1, a_{x x}=2, a_{x k}=1$, 
$a_{k}=1, a_{k k}=2$ and $\theta=0.5$. The time preference parameters are calibrated to fit true hyperbolic discounting with $d=0.7: \delta=.92671, \beta=.13683, \gamma=.48324$. Solving for the MPE strategy parameters gives us $\lambda_{3}=.075450, \mu_{3}=.008981$. The corresponding steady state addiction stock is $\bar{k}_{3}=.021154$. It implies steady state consumption $\bar{x}_{3}=.010577$.

\section{$9 \quad$ Further generalisations}

Our order-two and order-three quasi-hyperbolic specifications can be generalised further. This would enable us to attain an even better approximation to true hyperbolic discounting. However, the cost of achieving a better fit would be increased analytical and computational complexity.

To generate these discount functions, we use methods similar to those in Sections 3 and 4 . In particular, suppose that a discount function $D_{i}(\tau)$ yields lifetime payoffs that are amenable to recursive formulation. Consider the following two transformations:

$$
D_{2 i}(\tau)=\varepsilon D_{i}(\tau-1)
$$

and

$$
D_{3 i}(\tau)=D_{i}(\tau)+\varepsilon D_{3 i}(\tau-1)
$$

where $D_{2 i}(0)=1$ and $D_{3 i}(0)=1$. The resulting discount functions $D_{2 i}(\tau)$ and $D_{3 i}(\tau)$ will also give rise to lifetime payoffs that can be formulated recursively. Moreover, they will nest $D_{i}(\tau)$ as a special case which arises when $\varepsilon=1$ and $\varepsilon=0$, respectively. 


\subsection{Generalising order-two discounting}

\subsubsection{Definition}

Order-two quasi-hyperbolic discounting generalised Laibson's specifications by allowing an increasing effective discount factor for the first two adjacent trade-offs. We could extend this approach to three of these trade-offs by applying $(110)$ to $D_{2}(\tau)$. Specifically, consider a discount function $D_{22}(\tau)$ constructed as follows:

$$
D_{22}(0)=1, \quad D_{22}(\tau)=\varepsilon D_{2}(\tau-1), \tau \geqslant 1
$$

That is, $D_{22}(0)=1, D_{22}(1)=\varepsilon, D_{22}(2)=\varepsilon \beta \delta \gamma$, and $D_{22}(\tau)=\varepsilon \beta \gamma^{2} \delta^{\tau-1}$ for $\tau \geqslant 3$. The implied effective discount factors are i) $\varepsilon$ for the first adjacent trade-off, ii) $\beta \delta \gamma$ for the second adjacent trade-off, and $\delta \gamma$ for the third adjacent trade-off. For any other two consecutive future periods beyond $\tau=4$, the effective discount factor is always $\delta$. If $\beta<1, \gamma<1$ and $\varepsilon<\beta \gamma \delta$, impatience will be decreasing in the first three periods. Moreover, note that if $\gamma=1$, these preferences become identical to order-two quasi-hyperbolic discounting.

\subsubsection{Recursive formulation}

Using a method similar to that in Section 3, we can provide a recursive formulation for the lifetime payoff of a decision maker with such preferences. Let this lifetime payoff be denoted by $\Psi^{t}$ :

$$
\Psi^{t}=u^{t}+\varepsilon u^{t+1}+\varepsilon \beta \delta \gamma u^{t+2}+\varepsilon \beta \gamma^{2} \delta^{2} u^{t+3}+\varepsilon \beta \gamma^{2} \delta^{3} u^{t+4}+\varepsilon \beta \gamma^{2} \delta^{4} u^{t+5} \ldots
$$

It can be rewritten as

$$
\Psi^{t}=u^{t}+\varepsilon\left(u^{t+1}+\beta \delta \gamma u^{t+2}+\beta \gamma^{2} \delta^{2} u^{t+3}+\gamma \varepsilon^{2} \delta^{3} u^{t+4}+\beta \gamma^{2} \delta^{4} u^{t+5} \ldots\right)
$$


Define $U^{t+1}$ to be:

$$
U^{t+1}=u^{t+1}+\gamma \varepsilon \delta u^{t+2}+\gamma \varepsilon^{2} \delta^{2} u^{t+3}+\gamma \varepsilon^{2} \delta^{3} u^{t+4}+\ldots
$$

It follows that

$$
\Psi^{t}=u^{t}+\varepsilon U^{t+1}
$$

But $U^{t+1}$ is in fact the lifetime payoff of a decision maker with order-two quasi-hyperbolic discounting. From Section 3 we know that $U^{t+1}$ can be defined recursively as follows:

$$
\begin{aligned}
U^{t+1} & =\gamma \beta \delta V^{t+2}, \\
V^{t+2} & =\gamma \delta W^{t+3}, \\
W^{t+3} & =\delta W^{t+4} .
\end{aligned}
$$

\subsubsection{Generalised Euler equation}

Now consider the general intra-personal game as set up in Section 6. Using the above recursive formulation, we can derive an Euler equation for a decision maker with these preferences. His MPE strategy must solve the following Bellman equation:

$$
\Psi\left(k^{t}\right)=\max _{x^{t}}\left\{u\left(x^{t}, k^{t}\right)+\varepsilon U\left(a k^{t}+b x^{t}\right)\right\},
$$


where

$$
\begin{aligned}
U\left(k^{t}\right) & =u\left(h\left(k^{t}\right), k^{t}\right)+\gamma \beta \delta V\left(a k^{t}+b h\left(k^{t}\right)\right), \\
V\left(k^{t}\right) & =u\left(h\left(k^{t}\right), k^{t}\right)+\gamma \delta W\left(a k^{t}+b h\left(k^{t}\right)\right), \\
W\left(k^{t}\right) & =u\left(h\left(k^{t}\right), k^{t}\right)+\delta W\left(a k^{t}+b h\left(k^{t}\right)\right) .
\end{aligned}
$$

Differentiating (114) yields the first-order condition

$$
u_{x}^{t}+b \varepsilon_{k}^{t+1} U_{k}^{t+1}=0 .
$$

Solving this first-order condition for $U_{k}^{t+1}$ gives us $U_{k}^{t+1}=-u_{x}^{t} /(b \varepsilon)$. Next, differentiate (115) to get

$$
U_{k}^{t}=\left(h_{k}^{t} u_{x}^{t}+u_{k}^{t}\right)+\gamma \beta \delta\left(a+b h_{k}^{t}\right) V_{k}^{t+1} .
$$

Substituting $U_{k}^{t}$ above and solving for $V_{k}^{t+1}$, we obtain

$$
V_{k}^{t+1}=-\frac{u_{x}^{t-1}}{b \gamma \beta \delta \varepsilon\left(a+b h_{k}^{t}\right)}-\frac{\left(h_{k}^{t} u_{x}^{t}+u_{k}^{t}\right)}{\gamma \beta \delta\left(a+b h_{k}^{t}\right)} .
$$

Now differentiate (116):

$$
V_{k}^{t}=\left(h_{k}^{t} u_{x}^{t}+u_{k}^{t}\right)+\gamma \delta\left(a+b h_{k}^{t}\right) W_{k}^{t+1}=0
$$

Substitute $V_{k}^{t}$ in the above equation and solve for $W_{k}^{t+1}$ :

$$
W_{k}^{t+1}=-\frac{1}{\gamma \delta\left(a+b h_{k}^{t}\right)}\left[\frac{u_{x}^{t-2}}{b \gamma \beta \delta \varepsilon\left(a+b h_{k}^{t-1}\right)}+\frac{\left(h_{k}^{t-1} u_{x}^{t-1}+u_{k}^{t-1}\right)}{\gamma \beta \delta\left(a+b h_{k}^{t-1}\right)}+\left(h_{k}^{t} u_{x}^{t}+u_{k}^{t}\right)\right] .
$$


Finally, differentiating (117) delivers

$$
W_{k}^{t}=\left(h_{k}^{t} u_{x}^{t}+u_{k}^{t}\right)+\delta\left(a+b h_{k}^{t}\right) W_{k}^{t+1}=0
$$

Substituting $W_{k}^{t}, W_{k}^{t+1}$ in that condition gives us the decision maker's generalised Euler equation:

$$
\begin{gathered}
u_{x}^{t-2}+\varepsilon b\left(h_{k}^{t-1} u_{x}^{t-1}+u_{k}^{t-1}\right) \\
-b \gamma \beta \delta \varepsilon\left(a+b h_{k}^{t-1}\right)\left[\frac{u_{x}^{t-1}}{\gamma b \beta \varepsilon}-\left(1-\frac{1}{\gamma \beta}\right)\left(h_{k}^{t} u_{x}^{t}+u_{k}^{t}\right)+(1-\gamma) \delta\left(a+b h_{k}^{t}\right)\left(h_{k}^{t+1} u_{x}^{t+1}+u_{k}^{t+1}\right)\right]=0 .
\end{gathered}
$$

This equation can be used the compute the equilibrium strategies in our economic applications. Compared to (41), it has an extra term reflecting more complicated intra-personal strategic effects. This extra term increases the computational complexity when solving for the MPE strategy. Also, note that when $\gamma=1$, this Euler equation effectively reduces to (41).

\subsection{Combining the order-one and order-three specifications}

\subsubsection{Definition}

It is also possible to apply (110) to $D_{3}(\tau)$, thus effectively combining Laibson's approach with orderthree quasi-hyperbolic discounting. In particular, we could construct another discount function $D_{31}$ as follows:

$$
D_{31}(0)=1, \quad D_{31}(1)=\varepsilon, \quad D_{31}(\tau)=\varepsilon D_{3}(\tau-1)=\varepsilon\left(\frac{\beta \delta^{\tau}-\gamma^{\tau}+\delta(1-\beta) \gamma^{\tau-1}}{\delta-\gamma}\right), \tau \geqslant 2 .
$$

With this specification, the short-run discount factor for the trade-off between today and tomorrow is $\varepsilon$. Then, from next period onward, the discount factor becomes $D_{3}(\tau+1) / D_{3}(\tau)$. Impatience will be decreasing in all periods if $\varepsilon<\beta \delta+\gamma<\delta$. 


\subsubsection{Recursive formulation}

Again, the lifetime payoff of a decision maker with such preferences is amenable to recursive formulation. We denote this lifetime payoff by $\Psi^{t}$. By construction,

$$
\Psi^{t}=u^{t}+\varepsilon\left[D_{3}(0) u^{t+1}+D_{3}(1) u^{t+2}+D_{3}(2) u^{t+3}+\ldots\right] .
$$

Let $U^{t+1}=D_{3}(0) u^{t+1}+D_{3}(1) u^{t+2}+D_{3}(2) u^{t+3}+\ldots$ Therefore,

$$
\Psi^{t}=u^{t}+\varepsilon U^{t+1}
$$

Note that $U^{t+1}$ is the lifetime payoff of a period- $t+1$ decision maker with order-three quasihyperbolic preferences. In Section 4 we showed how to formulate $U^{t+1}$ recursively. Specifically,

$$
U^{t+1}=u^{t+1}+\gamma U^{t+2}+\beta \delta W^{t+2},
$$

where $W^{t+2}$ satisfies

$$
W^{t+2}=u^{t+2}+\delta W^{t+3} .
$$

\subsubsection{Generalised Euler equation}

Using this formulation, we can derive a generalised Euler equation for the MPE of the intra-personal game set up in Section 6. The equilibrium strategy solves the Bellman equation

$$
\Psi\left(k^{t}\right)=\max _{x^{t}}\left\{u\left(x^{t}, k^{t}\right)+\varepsilon U\left(a k^{t}+b x^{t}\right)\right\},
$$


where $U(k)$ satisfies

$$
\begin{aligned}
U\left(k^{t}\right) & =u\left(h\left(k^{t}\right), k^{t}\right)+\gamma U\left(a k^{t}+b h\left(k^{t}\right)\right)+\delta \beta W\left(a k^{t}+b h\left(k^{t}\right)\right), \\
W\left(k^{t}\right) & =u\left(h\left(k^{t}\right), k^{t}\right)+\delta W\left(a k^{t}+b h\left(k^{t}\right)\right) .
\end{aligned}
$$

Differentiating (120) yields the first-order condition

$$
u_{x}^{t}+\varepsilon b U_{k}^{t+1}=0
$$

Solving this condition for $U_{k}^{t+1}$ delivers $U_{k}^{t+1}=-u_{x}^{t} /(\varepsilon b)$. Next, differentiate (121) with respect to $k^{t}$ :

$$
U_{k}^{t}=h_{k}^{t} u_{x}^{t}+u_{k}^{t}+\left(a+b h_{k}^{t}\right)\left(\gamma U_{k}^{t+1}+\delta \beta W_{k}^{t+1}\right)
$$

Substitute $U_{k}^{t}, U_{k}^{t+1}$ in the above equation and solve for $W_{k}^{t+1}$ :

$$
W_{k}^{t+1}=-\frac{u_{x}^{t-1}}{\varepsilon b \delta \beta\left(a+b h_{k}^{t}\right)}-\frac{h_{k}^{t} u_{x}^{t}+u_{k}^{t}}{\delta \beta\left(a+b h_{k}^{t}\right)}+\frac{u_{x}^{t}}{\delta \beta \varepsilon b} .
$$

Finally, differentiate (122) with respect to $k^{t}$ to get

$$
W_{k}^{t}=h_{k}^{t} u_{x}^{t}+u_{k}^{t}+\delta\left(a+b h_{k}^{t}\right) W_{k}^{t+1}
$$

Substitution of $W_{k}^{t}, W_{k}^{t+1}$ delivers the agent's generalised Euler equation

$$
\begin{gathered}
u_{x}^{t-1}+\varepsilon b\left(h_{k}^{t} u_{x}^{t}+u_{k}^{t}\right) \\
-\left(a+b h_{k}^{t}\right)\left[u_{x}^{t}-\delta \varepsilon b \beta\left(1-\frac{1}{\beta}\right)\left(h_{k}^{t+1} u_{x}^{t+1}+u_{k}^{t+1}\right)+\delta u_{x}^{t}-\delta\left(a+b h_{k}^{t+1}\right) u_{x}^{t+1}\right]=0 .
\end{gathered}
$$


Given this equation, we can compute the agent's equilibrium strategies in the economic applications studied in Section 7.

\subsection{Generalising order-three discounting}

\subsubsection{Definition}

Our order-three quasi-hyperbolic specification defined the discount function through a second-order homogeneous difference equation. We could generalise this approach further by applying transformation (111) to $D_{3}(\tau)$. The resulting discount function will satisfy a higher order homogeneous difference equation. In particular, consider a discount function $D_{33}(\tau)$ constructed as follows:

$$
D_{33}(0)=1, \quad D_{33}(\tau+1)=D_{3}(\tau+1)+\varepsilon D_{33}(\tau), \tau \geqslant 1 .
$$

Thus, $D_{33}(0)=1, D_{33}(1)=\beta \delta+\gamma+\varepsilon, D_{33}(2)=\beta \delta^{2}+\gamma(\beta \delta+\gamma)+\varepsilon(\beta \delta+\gamma+\varepsilon), D_{33}(3)=$ $\left(\frac{\beta \delta^{4}-\gamma^{4}+\delta(1-\beta) \gamma^{3}}{\delta-\gamma}\right)+\varepsilon\left(\beta \delta^{2}+\gamma \beta \delta+\gamma^{2}\right)+\varepsilon^{2}(\beta \delta+\gamma+\varepsilon)$, etc. Note that when $\varepsilon=0$, this specification reduces to our order-three approximation.

To express $D_{33}(\tau)$ as a function of $\tau$ only, remember that $D_{3}(\tau)$ satisfies

$$
D_{3}(\tau+1)-\gamma D_{3}(\tau)=\delta\left[D_{3}(\tau)-\gamma D_{3}(\tau-1)\right] .
$$

Furthermore, the definition of $D_{33}(\tau)$ suggests that

$$
D_{3}(\tau+1)=D_{33}(\tau+1)-\varepsilon D_{33}(\tau) .
$$


Substitution into (123) delivers

$\left[D_{33}(\tau+1)-\varepsilon D_{33}(\tau)\right]-\gamma\left[D_{33}(\tau)-\varepsilon D_{33}(\tau-1)\right]=\delta\left\{\left[D_{33}(\tau)-\varepsilon D_{33}(\tau-1)\right]-\gamma\left[D_{33}(\tau-1)-\varepsilon D_{33}(\tau-2)\right]\right\}$,

which can be rearranged as

$$
D_{33}(\tau+1)-(\varepsilon+\gamma+\delta) D_{33}(\tau)+(\gamma \varepsilon+\delta \varepsilon+\delta \gamma) D_{33}(\tau-1)-\delta \gamma \varepsilon D_{33}(\tau-2)=0 .
$$

Condition (125) is a homogeneous difference equation of order three. Its characteristic equation,

$$
\chi^{3}-(\varepsilon+\gamma+\delta) \chi^{2}+(\gamma \varepsilon+\delta \varepsilon+\delta \gamma) \chi-\delta \gamma \varepsilon=0,
$$

has roots $\chi_{1}=\delta, \chi_{2}=\gamma, \chi_{3}=\varepsilon$. Therefore, the solution to the above difference equation is given by

$$
D_{33}(\tau)=c_{1} \delta^{\tau}+c_{2} \gamma^{\tau}+c_{3} \varepsilon^{\tau}
$$

The constants $c_{1}, c_{2}, c_{3}$ are chosen to match $D_{33}(1), D_{33}(2)$ and $D_{33}(3)$ :

$$
\begin{aligned}
c_{1} \delta+c_{2} \gamma+c_{3} \varepsilon & =\beta \delta+\gamma+\varepsilon \\
c_{1} \delta^{2}+c_{2} \gamma^{2}+c_{3} \varepsilon^{2} & =\beta \delta^{2}+\gamma \beta \delta+\gamma^{2}+\varepsilon(\beta \delta+\gamma+\varepsilon) . \\
c_{1} \delta^{3}+c_{2} \gamma^{3}+c_{3} \varepsilon^{3} & =\left(\frac{\beta \delta^{4}-\gamma^{4}+\delta(1-\beta) \gamma^{3}}{\delta-\gamma}\right)+\varepsilon\left(\beta \delta^{2}+\gamma \beta \delta+\gamma^{2}\right)+\varepsilon^{2}(\beta \delta+\gamma+\varepsilon) .
\end{aligned}
$$


Solving for $c_{1}, c_{2}, c_{3}$ gives us

$$
\left[\begin{array}{l}
c_{1} \\
c_{2} \\
c_{3}
\end{array}\right]=\left[\begin{array}{ccc}
\delta & \gamma & \varepsilon \\
\delta^{2} & \gamma^{2} & \varepsilon^{2} \\
\delta^{3} & \gamma^{3} & \varepsilon^{3}
\end{array}\right]^{-1}\left[\begin{array}{c}
\beta \delta+\gamma+\varepsilon \\
\beta \delta^{2}+\gamma \beta \delta+\gamma^{2}+\varepsilon(\beta \delta+\gamma+\varepsilon) \\
\left(\frac{\beta \delta^{4}-\gamma^{4}+\delta(1-\beta) \gamma^{3}}{\delta-\gamma}\right)+\varepsilon\left(\beta \delta^{2}+\gamma \beta \delta+\gamma^{2}\right)+\varepsilon^{2}(\beta \delta+\gamma+\varepsilon)
\end{array}\right]
$$

\subsubsection{Recursive formulation}

Next we provide a recursive representation of the decision maker's lifetime payoff in period $t$. Let it be denoted by $\Psi^{t}$ :

$$
\Psi^{t}=D_{33}(0) u^{t}+D_{33}(1) u^{t+1}+D_{33}(2) u^{t+2}+\ldots
$$

Similarly, in period $t+1$ we have that

$$
\Psi^{t+1}=D_{33}(0) u^{t+1}+D_{33}(1) u^{t+2}+D_{33}(2) u^{t+2}+\ldots
$$

Multiply (128) by $\varepsilon$ and subtract it from (127):

$\Psi^{t}-\varepsilon \Psi^{t+1}=u^{t}+\left[D_{33}(1)-\varepsilon D_{33}(0)\right] u^{t+1}+\left[D_{33}(2)-\varepsilon D_{33}(1)\right] u^{t+2}+\left[D_{33}(3)-\varepsilon D_{33}(2)\right] u^{t+3}+\ldots$

By construction, this expression is equivalent to

$$
\Psi^{t}=\varepsilon \Psi^{t+1}+u^{t}+D_{3}(1) u^{t+1}+D_{3}(2) u^{t+3}+\ldots
$$

Let $U^{t}=u^{t}+D_{3}(1) u^{t+1}+D_{3}(2) u^{t+3}+\ldots$. Therefore,

$$
\Psi^{t}=U^{t}+\varepsilon \Psi^{t+1}
$$


Note that $U^{t}$ is the lifetime payoff of a decision maker with order-three quasi-hyperbolic preferences.

In Section 4, we showed how to formulate $U^{t}$ recursively. It can be represented as

$$
\begin{aligned}
U^{t} & =u^{t}+\gamma U^{t+1}+\beta \delta W^{t+1}, \\
W^{t+1} & =u^{t+1}+\delta W^{t+2} .
\end{aligned}
$$

\subsubsection{Generalised Euler equation}

Now consider the general game of Section 6. We will derive an Euler equation for a decision maker with these preferences. The above recursive formulation of his lifetime payoff suggests that his MPE strategy solves the following Bellman equation:

$$
\Psi\left(k^{t}\right)=\max _{x^{t}}\left\{u\left(x^{t}, k^{t}\right)+\gamma U\left(a k^{t}+b x^{t}\right)+\delta \beta W\left(a k^{t}+b x^{t}\right)+\varepsilon \Psi\left(a k^{t}+b x^{t}\right)\right\},
$$

where

$$
\begin{aligned}
U\left(k^{t}\right) & =u\left(h\left(k^{t}\right), k^{t}\right)+\gamma U\left(a k^{t}+b h\left(k^{t}\right)\right)+\delta \beta W\left(a k^{t}+b h\left(k^{t}\right)\right), \\
W\left(k^{t}\right) & =u\left(h\left(k^{t}\right), k^{t}\right)+\delta W\left(a k^{t}+b h\left(k^{t}\right)\right) .
\end{aligned}
$$

The first-order and the envelope conditions associated with (129) are

$$
\begin{gathered}
u_{x}^{t}+b\left[\gamma U_{k}^{t+1}+\delta \beta W_{k}^{t+1}+\varepsilon \Psi_{k}^{t+1}\right]=0, \\
\Psi_{k}^{t}=u_{k}^{t}+a\left[\gamma U_{k}^{t+1}+\delta \beta W_{k}^{t+1}+\varepsilon \Psi_{k}^{t+1}\right] .
\end{gathered}
$$

From these equations we obtain

$$
\Psi_{k}^{t}=u_{k}^{t}-a u_{x}^{t} / b
$$


which implies that

$$
\gamma U^{t+1}+\delta \beta W^{t+1}=-u_{x}^{t} / b-\varepsilon\left(u_{k}^{t+1}-a u_{x}^{t+1} / b\right) .
$$

Differentiating (130) gives us

$$
U_{k}^{t}=h_{k}^{t} u_{x}^{t}+u_{k}^{t}+\left(a+b h_{k}^{t}\right)\left(\gamma U_{k}^{t+1}+\delta \beta W_{k}^{t+1}\right) .
$$

Substitute $\gamma U_{k}^{t+1}+\delta \beta W_{k}^{t+1}$ in this condition to obtain

$$
U_{k}^{t}=u_{k}^{t}-a u_{x}^{t} / b-\left(a+b h_{k}^{t}\right) \varepsilon\left(u_{k}^{t+1}-a u_{x}^{t+1} / b\right) .
$$

Now plug $U_{k}^{t}$ and $\Psi_{k}^{t}$ in the first-order condition and solve for $W_{k}^{t+1}$ :

$$
W_{k}^{t+1}=-\frac{u_{x}^{t}+b(\varepsilon+\gamma)\left(u_{k}^{t+1}-a u_{x}^{t+1} / b\right)-b\left(a+b h_{k}^{t+1}\right) \varepsilon\left(u_{k}^{t+2}-a u_{x}^{t+2} / b\right)}{b \delta \beta}
$$

Finally, differentiating (131) yields

$$
W_{k}^{t}=h_{k}^{t} u_{x}^{t}+u_{k}^{t}+\delta\left(a+b h_{k}^{t}\right) W_{k}^{t+1}
$$

Substituting $W_{k}^{t}, W_{k}^{t+1}$ delivers the decision maker's generalised Euler equation:

$$
\begin{gathered}
u_{x}^{t}+b \delta \beta u_{k}^{t+1}-\delta\left[a+b(1-\beta) h_{k}^{t}\right] u_{x}^{t+1}-(\varepsilon+\gamma)\left[a u_{x}^{t+1}-b u_{k}^{t+1}\right] \\
-\left(a+b h_{k}^{t+1}\right)\left\{[\delta b(\varepsilon+\gamma)+\varepsilon]\left(u_{k}^{t+2}-a u_{x}^{t+2} / b\right)-b \delta\left(a+b h_{k}^{t+2}\right) \varepsilon\left(u_{k}^{t+3}-a u_{x}^{t+3} / b\right)\right\}=0 .
\end{gathered}
$$

This condition can be used to compute the MPE strategies in our economic applications. 


\subsection{Combining the order-two and order-three specifications}

\subsubsection{Definition}

We can also construct a combination of our order-two and order-three specifications by applying transformation (111) to $D_{2}(\tau)$. This combined approach would nest both our order-two and orderthree approximations as special cases. Thus, a calibration of this specification will necessarily deliver a better fit to true hyperbolic discounting than both $D_{2}(\tau)$ and $D_{3}(\tau)$.

Specifically, define the discount function $D_{32}(\tau)$ as follows

$$
D_{32}(0)=1, \quad D_{32}(\tau+1)=D_{2}(\tau+1)+\varepsilon D_{32}(\tau), \forall \tau \geqslant 2,
$$

where $D_{2}(\tau)$ is our order-two generalisation as defined in Section 3. This definition yields $D_{32}(0)=$ $1, D_{32}(1)=\beta \delta \gamma+\varepsilon, D_{32}(2)=\beta \delta^{2} \gamma^{2}+\varepsilon(\beta \delta \gamma+\varepsilon), D_{32}(3)=\beta \gamma^{2} \delta^{3}+\varepsilon\left(\beta \delta^{2} \gamma^{2}+\varepsilon(\beta \delta \gamma+\varepsilon)\right)$. Note that if $\varepsilon=0, D_{32}(\tau)$ is reduced to our order-two approximation. If, on the other hand $\gamma=1$, $D_{2}(\tau)$ is just the order-one quasi-hyperbolic discount function, so $D_{32}(\tau)$ becomes equivalent to the order-three approximation.

This discount function is defined by the same third-order difference equation as $D_{33}(\tau)$. However, the initial conditions differ from those in the previous subsection. Therefore,

$$
D_{32}(\tau)=\tilde{c}_{1} \delta^{\tau}+\tilde{c}_{2} \gamma^{\tau}+\tilde{c}_{3} \varepsilon^{\tau}
$$


where the constants $\tilde{c}_{1}, \tilde{c}_{2}, \tilde{c}_{3}$ now satisfy

$$
\begin{aligned}
\tilde{c}_{1} \delta+\tilde{c}_{2} \gamma+\tilde{c}_{3} \varepsilon & =\beta \delta \gamma+\varepsilon \\
\tilde{c}_{1} \delta^{2}+\tilde{c}_{2} \gamma^{2}+\tilde{c}_{3} \varepsilon^{2} & =\beta \delta^{2} \gamma^{2}+\varepsilon(\beta \delta \gamma+\varepsilon), \\
\tilde{c}_{1} \delta^{3}+\tilde{c}_{2} \gamma^{3}+\tilde{c}_{3} \varepsilon^{3} & =\beta \gamma^{2} \delta^{3}+\varepsilon\left(\beta \delta^{2} \gamma^{2}+\varepsilon(\beta \delta \gamma+\varepsilon)\right) .
\end{aligned}
$$

Solving (133), (134), (135) gives us

$$
\left[\begin{array}{l}
c_{1} \\
c_{2} \\
c_{3}
\end{array}\right]=\left[\begin{array}{ccc}
\delta & \gamma & \varepsilon \\
\delta^{2} & \gamma^{2} & \varepsilon^{2} \\
\delta^{3} & \gamma^{3} & \varepsilon^{3}
\end{array}\right]^{-1}\left[\begin{array}{c}
\beta \delta \gamma+\varepsilon \\
\beta \delta^{2} \gamma^{2}+\varepsilon(\beta \delta \gamma+\varepsilon) \\
\gamma^{2} \delta^{3}+\varepsilon\left(\beta \delta^{2} \gamma^{2}+\varepsilon(\beta \delta \gamma+\varepsilon)\right)
\end{array}\right]
$$

\subsubsection{Recursive formulation}

To provide a recursive formulation of the decision maker's lifetime payoff, we follow the same method as in the previous subsection. Let this lifetime payoff from the period- $t$ perspective be $\Psi^{t}$ :

$$
\Psi^{t}=D_{32}(0) u^{t}+D_{32}(1) u^{t+1}+D_{32}(2) u^{t+2}+\ldots
$$

In the subsequent period, we will have

$$
\Psi^{t+1}=D_{32}(0) u^{t+1}+D_{32}(1) u^{t+2}+D_{32}(2) u^{t+2}+\ldots
$$

Multiply (136) by $\varepsilon$ and subtract from (137) to get

$\Psi^{t}-\varepsilon \Psi^{t+1}=u^{t}+\left[D_{32}(1)-\varepsilon D_{32}(0)\right] u^{t+1}+\left[D_{32}(2)-\varepsilon D_{32}(1)\right] u^{t+2}+\left[D_{32}(3)-\varepsilon D_{32}(2)\right] u^{t+3}+\ldots$ 
By construction, the above equation is identical to

$$
\Psi^{t}-\varepsilon \Psi^{t+1}=u^{t}+D_{2}(1) u^{t+1}+D_{2}(2) u^{t+2}+D_{2}(3) u^{t+3}+\ldots
$$

But the right-hand side of (138) is the lifetime payoff of a decision maker with order-two quasihyperbolic preferences. Its recursive representation was derived in Section 3. In particular, let $U^{t}=u^{t}+D_{2}(1) u^{t+1}+D_{2}(2) u^{t+2}+D_{2}(3) u^{t+3}+\ldots$ Then we have that

$$
\Psi^{t}=U^{t}+\varepsilon \Psi^{t+1}
$$

where

$$
\begin{aligned}
U^{t} & =u^{t}+\beta \delta \gamma V^{t+1}, \\
V^{t+1} & =u^{t+1}+\gamma \delta W^{t+2}, \\
W^{t+2} & =u^{t+2}+\delta W^{t+3} .
\end{aligned}
$$

\subsubsection{Generalised Euler equation}

Consider the general game of Section 6. The MPE strategy of the decision maker will solve the following Bellman equation:

$$
\Psi\left(k^{t}\right)=\max _{x^{t}}\left\{u\left(x^{t}, k^{t}\right)+\gamma \delta \beta V\left(a k^{t}+b x^{t}\right)+\varepsilon \Psi\left(a k^{t}+b x^{t}\right)\right\} .
$$


The value functions $W, \Omega$ must satisfy the same functional equations as (36), (37):

$$
\begin{aligned}
V\left(k^{t}\right) & =u\left(h\left(k^{t}\right), k^{t}\right)+\gamma \delta W\left(a k^{t}+b h\left(k^{t}\right)\right), \\
W\left(k^{t}\right) & =u\left(h\left(k^{t}\right), k^{t}\right)+\delta W\left(a k^{t}+b h\left(k^{t}\right)\right) .
\end{aligned}
$$

Now let us derive the corresponding Euler equation. Differentiating (142) yields a first-order condition and an envelope condition:

$$
\begin{gathered}
u_{x}^{t}+b\left[\gamma \delta \beta V_{k}^{t+1}+\varepsilon \Psi_{k}^{t+1}\right]=0 \\
\Psi_{k}^{t}=u_{k}^{t}+a\left[\gamma \delta \beta V_{k}^{t+1}+\varepsilon \Psi_{k}^{t+1}\right],
\end{gathered}
$$

respectively. These conditions imply that

$$
\Psi_{k}^{t}=u_{k}^{t}-a u_{x}^{t} / b
$$

Substituting $\Psi_{k}^{t}$ in the first-order condition gives us

$$
V_{k}^{t+1}=-\frac{u_{x}^{t}+\varepsilon\left(b u_{k}^{t+1}-a u_{x}^{t+1}\right)}{b \gamma \delta \beta} .
$$

Next differentiate (143) to obtain

$$
V_{k}^{t}=\left(h_{k}^{t} u_{x}^{t}+u_{k}^{t}\right)+\gamma \delta\left(a+b h_{k}^{t}\right) W_{k}^{t+1}=0
$$

Substitute $V_{k}^{t}$ and solve for $W_{k}^{t+1}$ :

$$
W_{k}^{t+1}=-\frac{1}{b \delta \gamma^{2} \delta^{2}\left(a+b h_{k}^{t}\right)}\left[u_{x}^{t-1}+\varepsilon\left(b u_{k}^{t}-a u_{x}^{t}\right)+b \gamma \delta \beta\left(h_{k}^{t} u_{x}^{t}+u_{k}^{t}\right)\right]
$$


Finally, differentiate (144):

$$
W_{k}^{t}=\left(h_{k}^{t} u_{x}^{t}+u_{k}^{t}\right)+\delta\left(a+b h_{k}^{t}\right) W_{k}^{t+1}=0
$$

Substituting $W_{k}^{t}, W_{k}^{t+1}$ in the above condition yields the decision maker's generalised Euler equation:

$$
\begin{gathered}
u_{x}^{t-1}+\varepsilon\left(b u_{k}^{t}-a u_{x}^{t}\right)+b \gamma \delta \beta\left(h_{k}^{t} u_{x}^{t}+u_{k}^{t}\right)+b \delta \gamma^{2} \delta^{2}\left(a+b h_{k}^{t}\right)\left(h_{k}^{t} u_{x}^{t}+u_{k}^{t}\right) \\
-\delta\left(a+b h_{k}^{t}\right)\left[u_{x}^{t}+\varepsilon\left(b u_{k}^{t+1}-a u_{x}^{t+1}\right)+b \gamma \delta \beta\left(h_{k}^{t+1} u_{x}^{t+1}+u_{k}^{t+1}\right)\right]=0 .
\end{gathered}
$$

Again, we can use this Euler equation to compute the MPE strategy in the economic applications.

\section{Conclusion}

This paper studies generalisations of quasi-hyperbolic discounting which yield lifetime payoffs that are amenable to recursive representation. Our proposals nest Laibson's $(\beta, \delta)$-preferences as a special case. Consequently, they always provide better approximations to true hyperbolic discounting. We illustrate this point by undertaking a calibration exercise. It clearly shows that our order-two and order-three specifications deliver a superior goodness of fit as compared to standard quasihyperbolic discounting.

We also investigate how these approximations affect the predictions of two popular economic models: i) Laibson's consumption-saving model, and ii) smoking and internality taxes. Specifically, we analyse a general intra-personal game which includes these models as special cases. The analysis is then used to compute the equilibrium for true hyperbolic discounters, and compare it to those for agents with order-two and order-three quasi-hyperbolic discounting. Again, our numerical examples 
demonstrate that order-three (and, to a lesser extent, order-two) discounting yield policies that are close to those with true hyperbolic discounting.

In the aforementioned applications, we can characterise the behaviour of true hyperbolic agents without resorting to approximations of their time preferences. However, there are settings where exact solutions to these problems are not available. We consider two such settings, featuring i) Markovian uncertainty, and ii) linear-quadratic utility. We argue that in these cases our proposals are quite compelling: they yield finite lifetime payoffs, and can be analysed with standard dynamic programming techniques.

Finally, we show that our order-two and order-three quasi-hyperbolic specifications can be generalised further. These higher-order generalisations would allow us to obtain even closer approximations to true hyperbolic discounting. We provide expressions for their discount functions, and derive equilibrium conditions for the general intra-personal game. 


\section{References}

[1] Ainslie, G., 1975, Specious Reward: A Behavioral Theory of Impulsiveness and Impulse Contol, Psychological Bulletin 82(4), 463-496.

[2] Ainslie, G., 1992, Picoeconomics, Cambridge: Cambridge University Press.

[3] Ainslie, G and R. Herrnstein, 1981, Preference Reversal and Delayed Reinforcement, Animal Learning and Behavior 9(4), 476-482.

[4] Barro, R., 1999, Ramsey Meets Laibson in the Neoclassical Growth Model, Quarterly Journal of Economics 114(4), 1125-1152.

[5] Becker, G and K. Murphy, 1988, A Theory of Rational Addiction, Journal of Political Economy 96, 675-700.

[6] Bellman, R., 1952, On the Theory of Dynamic Programming, Proceedings of the National Academy of Sciences of the United States of America 38(8), 716-719.

[7] Calcott, P and V. Petkov, 2015, Consumption Models with Homogeneous Utility and Generalised Hyperbolic Discounting, Working Paper.

[8] Christensen-Szalanski, J., 1984, Discount Functions and the Measurement of Patients' Values. Women's Decisions During Childbirth, Medical Decision Making 4(1), 47-58.

[9] Chung, S-H and R. Herrnstein, 1961, Relative and Absolute Strengths of Response as a Function of Frequency of Reinforcement, Journal of the Experimental Analysis of Animal Behavior 4, 267-272.

[10] Diamond, P and B. Koszegi, 2003, Quasi-hyperbolic Discounting and Retirement, Journal of Public Economics 87, 1839-1872. 
[11] Frederick, S., G. Loewenstein, and T.O'Donoghue, 2002, Time Discounting and Time Preference: A Critical Review, Journal of Economic Literature 40(2), 351-401.

[12] Green, L., A. Fry, and J. Myerson, 1994, Discounting of Delayed Rewards: A Life-Span Comparison, Psychological Science 5(1), 33-36.

[13] Gruber, J and B. Koszegi, 2001, Is Addiction "Rational"?, Quarterly Journal of Economics 116(4), 1261-1305.

[14] Kocherlakota, N., 2001, Looking for Evidence of Time-Inconsistent Preferences in Asset Market Data, Federal Reserve Bank of Minneapolis Quarterly Review 25(3), 13-24.

[15] Laibson, D., 1997, Golden Eggs and Hyperbolic Discounting, Quarterly Journal of Economics $112(2), 443-477$.

[16] Laibson, D., 1998, Life-cycle consumption and hyperbolic discount functions, European Economic Review, 42(3-5), 861-871.

[17] Loewenstein, G and D. Prelec, 1992, Anomalies in Intertemporal Choice: Evidence and Interpretation, Quarterly Journal of Economics 107(2), 573-597.

[18] Mazur, J., 1987, An Adjusting Procedure for Studying Delayed Reinforcement in Quantitative Analyses of Behavior 5, 55-73.

[19] Millar, A and D. Navarick, 1984, Self-control and Choice in Humans: Effects of Video Game Playing as a Positive Reinforcer, Learning and Motivation 15, 203-218.

[20] O’Donoghue, T and M.Rabin, 1999, Doing It Now or Later, American Economic Review 89(1), 103-124. 
[21] Paserman, M., 2008, Job Search and Hyperbolic Discounting: Structural Estimation and Policy Evaluation, Economic Journal 118(531), 1418-1452.

[22] Phelps, E and R. Pollak, 1968, On Second-best National Saving and Game Equilibrium Growth, Review of Economic Studies 35(2), 185-199.

[23] Samuelson, P., 1937, A Note on Measurement of Utility, Review of Economic Studies 4(2), $155-161$.

[24] Strotz, R., 1955-1956, Myopia and Inconsistency in Dynamic Utility Maximization, The Review of Economic Studies 23(3), 165-180.

[25] Thaler, R., 1981, Some Empirical Evidence on Dynamic Inconsistency, Economics Letters 8, 201-207. 


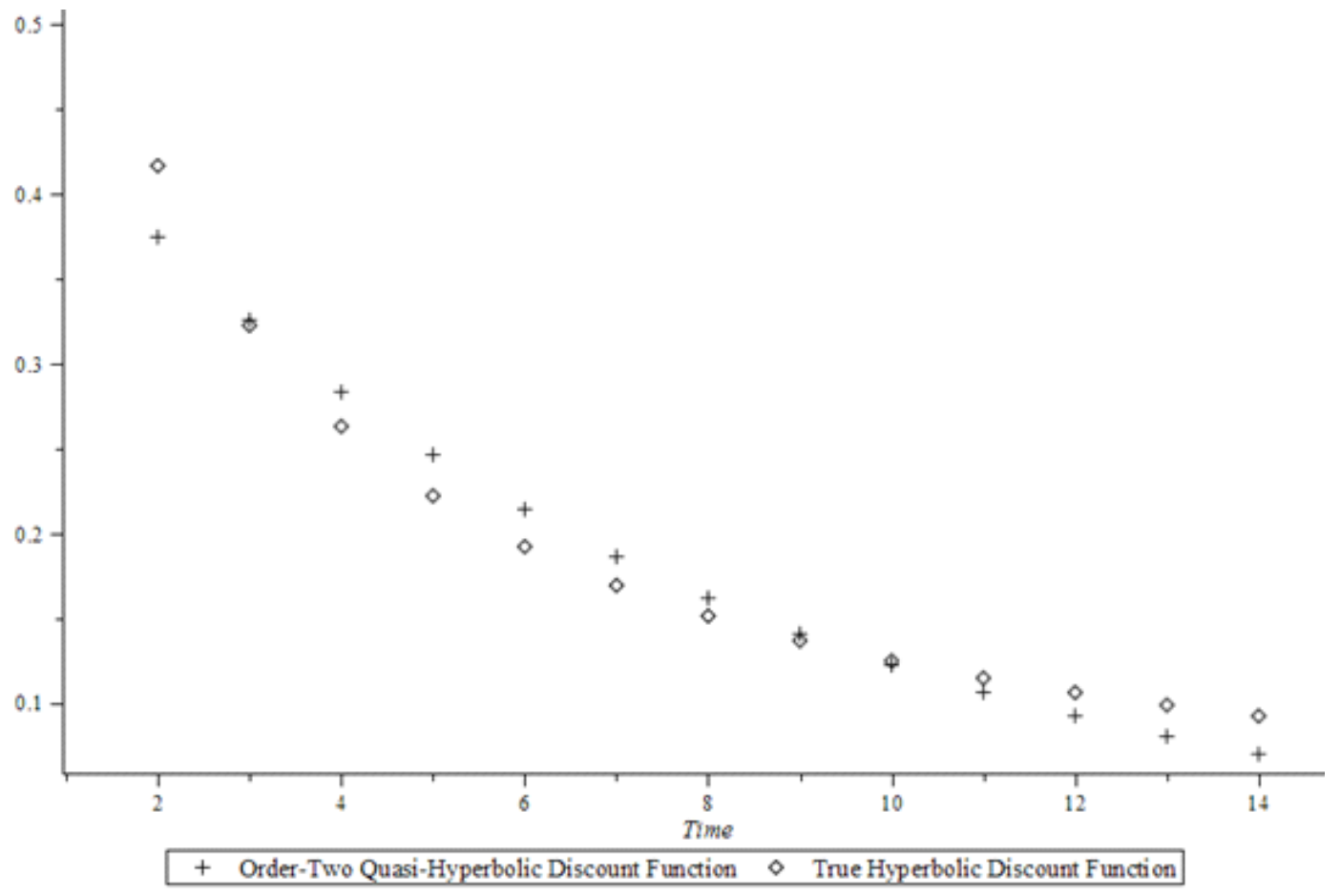

Figure 1a

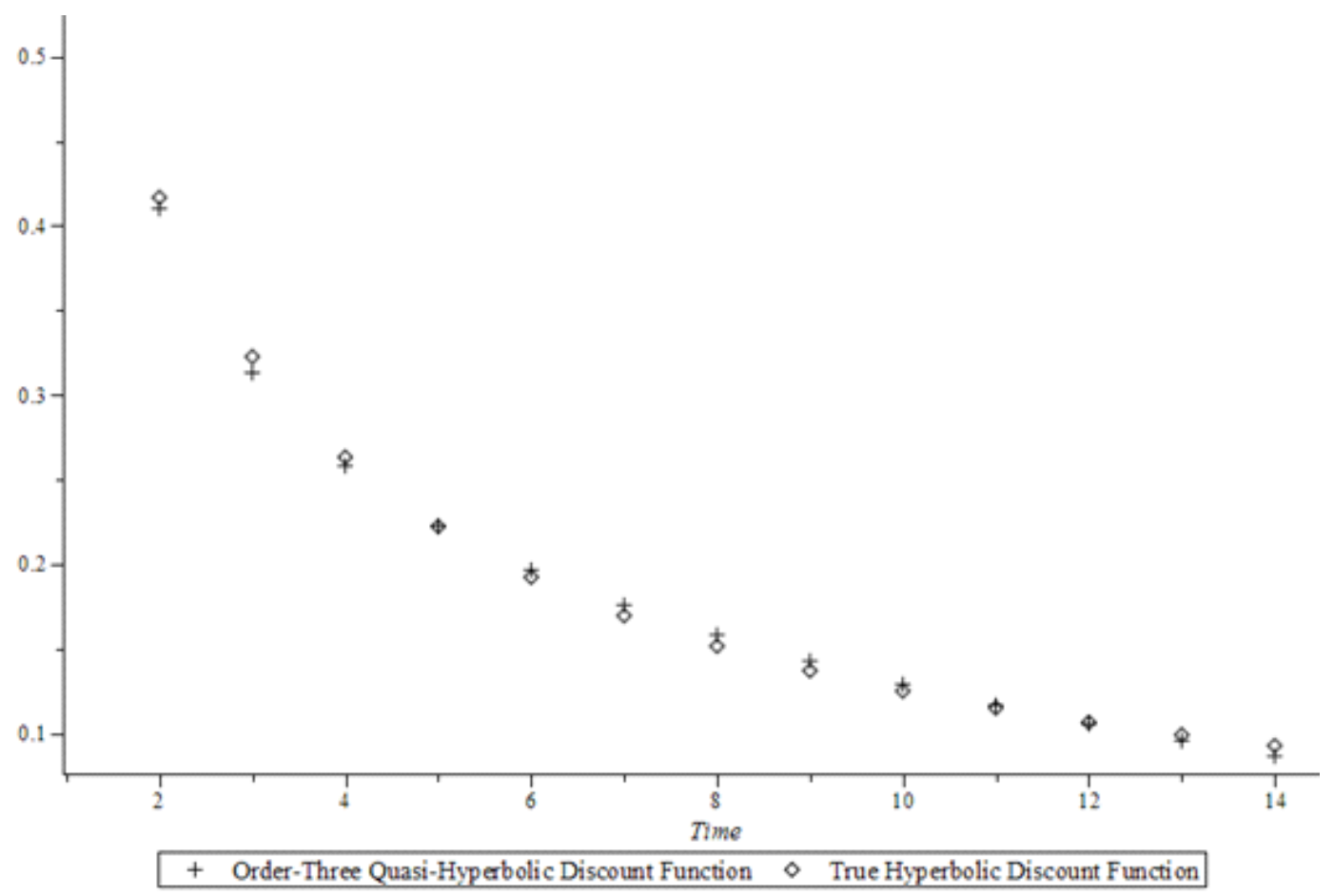

Figure 1b 


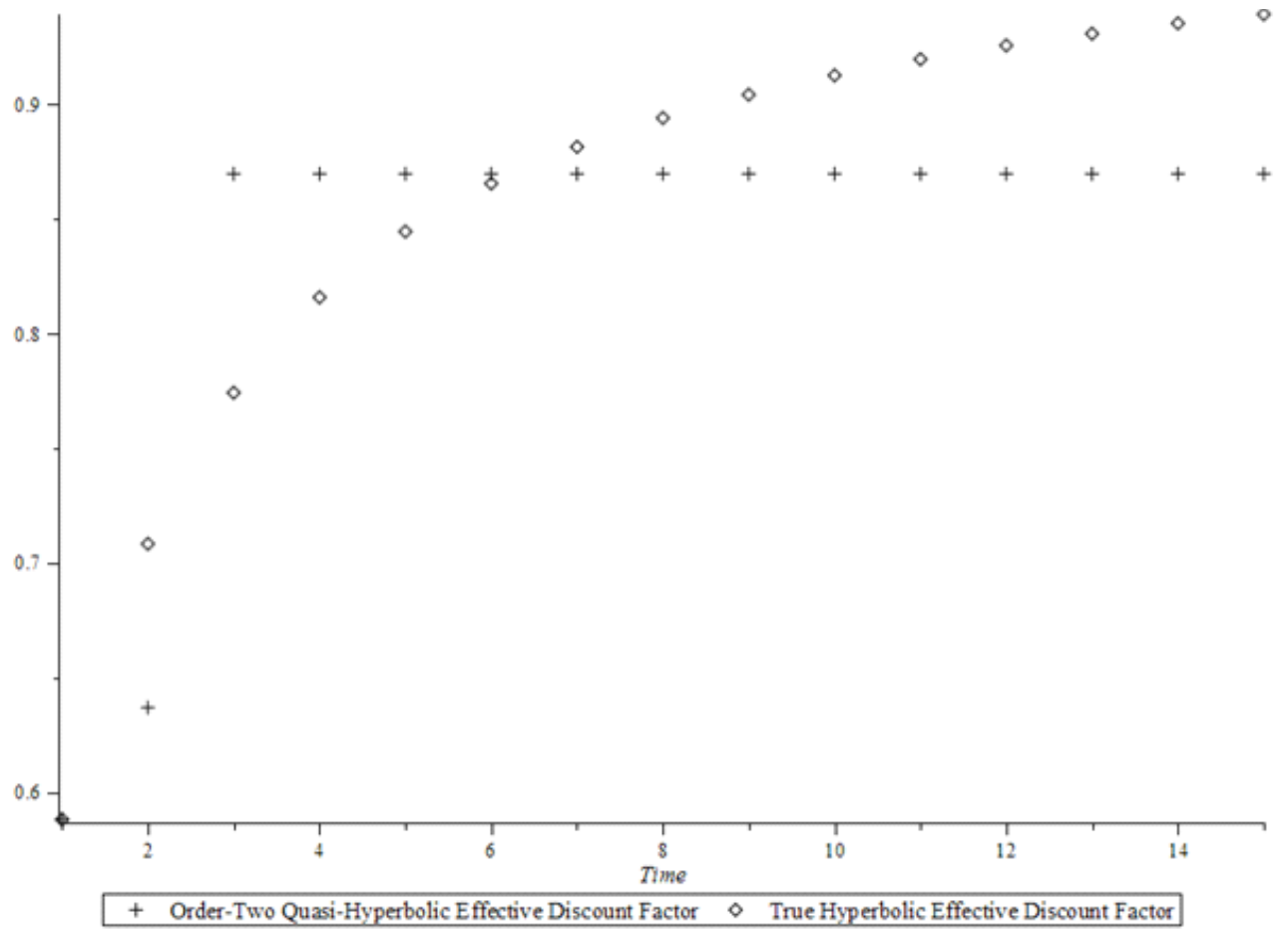

Figure 2a

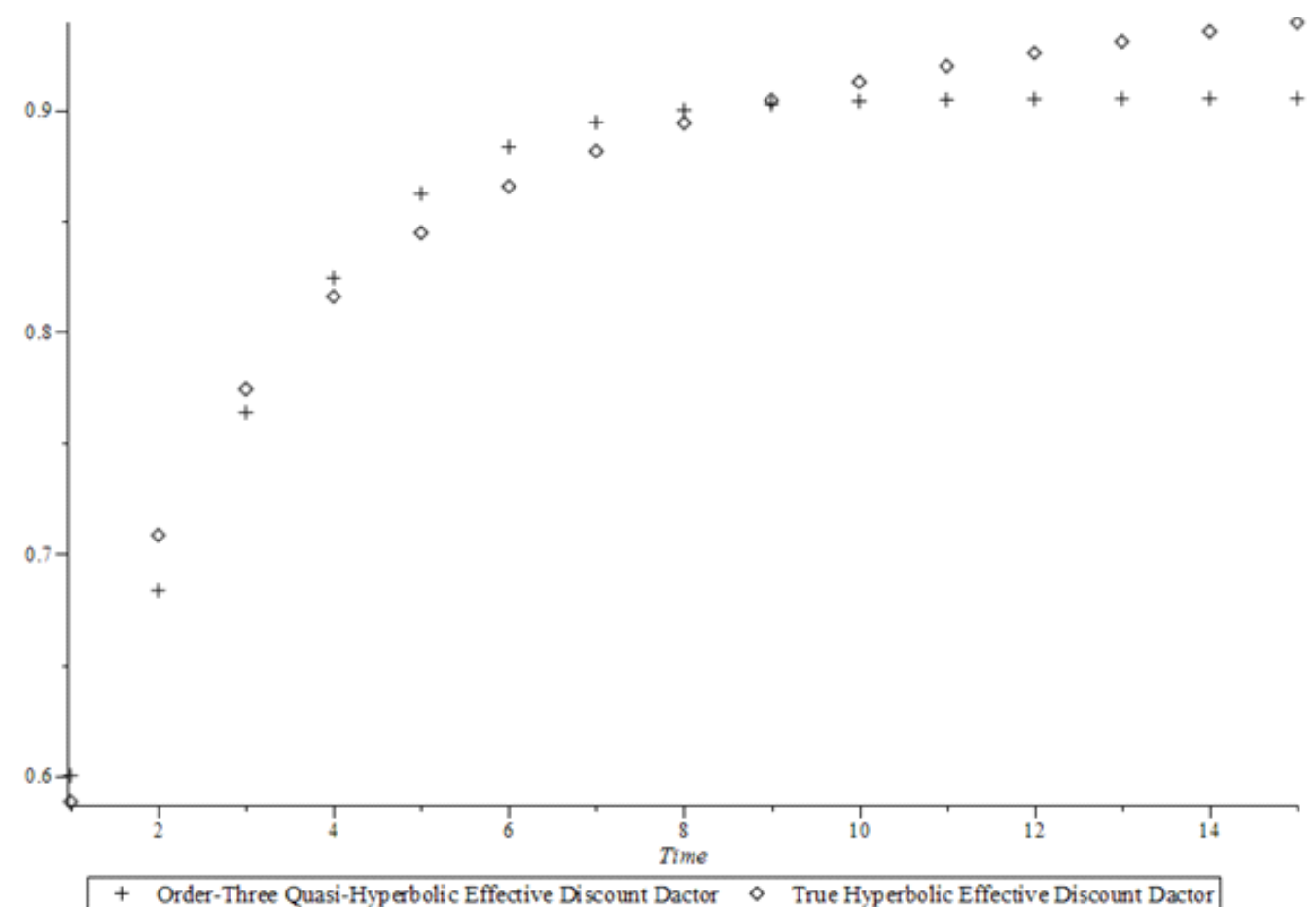

Figure $2 b$ 


\begin{tabular}{|l|l|l|l|l|l|}
\hline$D_{h}(t)$ & $d=.3$ & $d=.5$ & $d=.7$ & $d=.8$ & $d=.9$ \\
\hline & $\beta^{*}=.72017$ & $\beta^{*}=.62576$ & $\beta^{*}=.56111$ & $\beta^{*}=.53521$ & $\beta^{*}=.51228$ \\
O1 QH & $\delta^{*}=.90934$ & $\delta^{*}=.88378$ & $\delta^{*}=.86393$ & $\delta^{*}=.85536$ & $\delta^{*}=.84749$ \\
& $L_{1}^{*}=.04115$ & $L_{1}^{*}=.04019$ & $L_{1}^{*}=.03468$ & $L_{1}^{*}=.03182$ & $L_{1}^{*}=.02912$ \\
\hline \multirow{2}{*}{ O2 QH } & $\gamma^{*}=.77045$ & $\gamma^{*}=.70620$ & $\gamma^{*}=.66703$ & $\gamma^{*}=.65258$ & $\gamma^{*}=.64043$ \\
& $\beta^{*}=1.08596$ & $\beta^{*}=1.04537$ & $\beta^{*}=.98796$ & $\beta^{*}=.95801$ & $\beta^{*}=.92838$ \\
& $\delta_{2}^{*}=.91938$ & $\delta^{*}=.90306$ & $\delta^{*}=.89261$ & $\delta^{*}=.88863$ & $\delta^{*}=.88521$ \\
& $\gamma^{*}=.66772$ & $\gamma^{*}=.56296$ & $\gamma^{*}=.48324$ & $\gamma^{*}=.45006$ & $\gamma^{*}=.42047$ \\
& $\beta^{*}=.12108$ & $\beta^{*}=.13205$ & $\beta^{*}=.13683$ & $\beta^{*}=.13787$ & $\beta^{*}=.13826$ \\
O3 QH & $\delta^{*}=.94872$ & $\delta^{*}=.93610$ & $\delta^{*}=.92671$ & $\delta^{*}=.92274$ & $\delta^{*}=.91914$ \\
& $L_{3}^{*}=.00069$ & $L_{3}^{*}=.00140$ & $L_{3}^{*}=.00184$ & $L_{3}^{*}=.00196$ & $L_{3}^{*}=.00204$ \\
\hline
\end{tabular}

Table 1: Parameter Calibration 


\begin{tabular}{|l|l|l|l|l|l|}
\hline$D_{h}(t)$ & $d=.3$ & $d=.5$ & $d=.7$ & $d=.8$ & $d=.9$ \\
\hline & $\beta^{*}=.78888$ & $\beta^{*}=.72132$ & $\beta^{*}=.66865$ & $\beta^{*}=.64519$ & $\beta^{*}=.62317$ \\
O1 QH & $\delta^{*}=.89306$ & $\delta^{*}=.85205$ & $\delta^{*}=.81914$ & $\delta^{*}=.80527$ & $\delta^{*}=.79287$ \\
& $\tilde{L}_{1}^{*}=.01278$ & $\tilde{L}_{1}^{*}=.00887$ & $\tilde{L}_{1}^{*}=.00588$ & $\tilde{L}_{1}^{*}=.00483$ & $\tilde{L}_{1}^{*}=.00400$ \\
\hline & $\gamma^{*}=.82264$ & $\gamma^{*}=.77775$ & $\gamma^{*}=.749837$ & $\gamma^{*}=.73927$ & $\gamma^{*}=.73023$ \\
& $\beta^{*}=1.02895$ & $\beta^{*}=.96875$ & $\beta^{*}=.90337$ & $\beta^{*}=.87183$ & $\beta^{*}=.841608$ \\
& $\delta^{*}=.90876$ & $\delta^{*}=.88482$ & $\delta^{*}=.86840$ & $\delta^{*}=.86197$ & $\delta^{*}=.85640$ \\
& $\tilde{L}_{2}^{*}=.00586$ & $\tilde{L}_{2}^{*}=.00345$ & $\tilde{L}_{2}^{*}=.00207$ & $\tilde{L}_{2}^{*}=.00164$ & $\tilde{L}_{2}^{*}=.00132$ \\
\hline \multirow{2}{*}{$\mathrm{O} 3 \mathrm{QH}$} & $\gamma^{*}=.64775$ & $\gamma^{*}=.52910$ & $\gamma^{*}=.44093$ & $\gamma^{*}=.40531$ & $\gamma^{*}=.37420$ \\
& $\beta^{*}=.13787$ & $\beta^{*}=.15985$ & $\beta^{*}=.17202$ & $\beta^{*}=.175568$ & $\beta^{*}=.17783$ \\
& $\delta^{*}=.94446$ & $\delta^{*}=.92757$ & $\delta^{*}=.91389$ & $\delta^{*}=.90787$ & $\delta^{*}=.90230$ \\
& $\tilde{L}_{3}^{*}=.00022$ & $\tilde{L}_{3}^{*}=.00033$ & $\tilde{L}_{3}^{*}=.00034$ & $\tilde{L}_{3}^{*}=.00032$ & $\tilde{L}_{3}^{*}=.00030$ \\
\hline
\end{tabular}

Table 2: Parameter Calibration

\begin{tabular}{|l|l|l|l|l|l|}
\hline Value of $d$ in $D_{h}(t)$ & $d=.3$ & $d=.5$ & $d=.7$ & $d=.8$ & $d=.9$ \\
\hline True hyperbolic & $\lambda_{h}=.44289$ & $\lambda_{h}=.65019$ & $\lambda_{h}=.77144$ & $\lambda_{h}=.81250$ & $\lambda_{h}=.84473$ \\
\hline O1 QH & $\lambda_{1}=.51299$ & $\lambda_{1}=.75428$ & $\lambda_{1}=.85732$ & $\lambda_{1}=.88671$ & $\lambda_{1}=.90819$ \\
\hline O2 QH & $\lambda_{2}=.47243$ & $\lambda_{2}=.68079$ & $\lambda_{2}=.79135$ & $\lambda_{2}=.82974$ & $\lambda_{2}=.85663$ \\
\hline O3 QH & $\lambda_{3}=.43584$ & $\lambda_{3}=.63647$ & $\lambda_{3}=.75680$ & $\lambda_{3}=.79866$ & $\lambda_{3}=.83205$ \\
\hline
\end{tabular}

Table 3: Laibson's Consumption-Saving Model

\begin{tabular}{|l|l|l|l|l|l|}
\hline Value of $d$ in $D_{h}(t)$ & $d=.3$ & $d=.5$ & $d=.7$ & $d=.8$ & $d=.9$ \\
\hline True hyperbolic & $\psi_{h}=.28056$ & $\psi_{h}=.48041$ & $\psi_{h}=.61902$ & $\psi_{h}=.67400$ & $\psi_{h}=.72201$ \\
\hline O1 QH & $\psi_{1}=.37039$ & $\psi_{1}=.55800$ & $\psi_{1}=.68219$ & $\psi_{1}=.73078$ & $\psi_{1}=.77313$ \\
\hline O2 QH & $\psi_{2}=.31644$ & $\psi_{2}=.51128$ & $\psi_{2}=.64507$ & $\psi_{2}=.69801$ & $\psi_{2}=.74423$ \\
\hline O3 QH & $\psi_{3}=.26888$ & $\psi_{3}=.46763$ & $\psi_{3}=.60855$ & $\psi_{3}=.66502$ & $\psi_{3}=.71452$ \\
\hline
\end{tabular}

Table 4: Internality Taxes 


\begin{tabular}{|c|c|c|c|c|}
\hline & $p_{s}=0.6$ & $p_{s}=0.7$ & $p_{s}=0.8$ & $p_{s}=0.9$ \\
\hline & $q_{s}=0.4$ & $q_{s}=0.3$ & $q_{s}=0.2$ & $q_{s}=0.1$ \\
\hline$\lambda_{s}$ & .042347 & .047457 & .055683 & .07103 \\
\hline$\lambda_{b}$ & .035832 & .032508 & .028875 & .02490 \\
\hline
\end{tabular}

Table 5: Optimal Policies with Markovian Uncertainty

\begin{tabular}{|c|c|c|c|c|}
\hline & $p_{s}=0.6$ & $p_{s}=0.7$ & $p_{s}=0.8$ & $p_{s}=0.9$ \\
\hline & $q_{s}=0.4$ & $q_{s}=0.3$ & $q_{s}=0.2$ & $q_{s}=0.1$ \\
\hline$\lambda_{s}$ & .173622 & .197130 & .232089 & .289212 \\
\hline$\lambda_{b}$ & .142086 & .127444 & .112715 & .097869 \\
\hline$\psi_{s}$ & 1.20654 & 1.14610 & 1.05950 & .930375 \\
\hline$\psi_{b}$ & 1.29205 & 1.35063 & 1.43263 & 1.55097 \\
\hline
\end{tabular}

Table 6: Equilibrium Strategies and Optimal Tax Rates with Markovian Uncertainty (Order-Two Discounting)

\begin{tabular}{|c|c|c|c|c|}
\hline & $p_{s}=0.6$ & $p_{s}=0.7$ & $p_{s}=0.8$ & $p_{s}=0.9$ \\
\hline & $q_{s}=0.4$ & $q_{s}=0.3$ & $q_{s}=0.2$ & $q_{s}=0.1$ \\
\hline$\lambda_{s}$ & .164293 & .187722 & .223735 & .284507 \\
\hline$\lambda_{b}$ & 133937 & .119758 & .105426 & .090993 \\
\hline$\psi_{s}$ & 1.15220 & 1.09606 & 1.01516 & .893176 \\
\hline$\psi_{b}$ & 1.23201 & 1.28651 & 1.36329 & 1.47554 \\
\hline
\end{tabular}

Table 7: Equilibrium Strategies and Optimal Tax Rates with Markovian Uncertainty (Order-Three Discounting) 